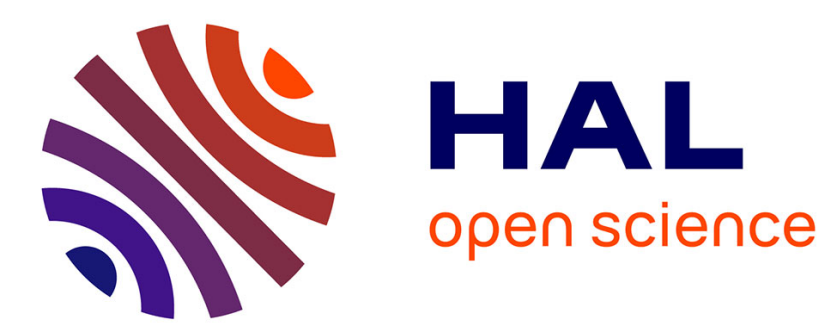

\title{
Un lot d'archives inédit de Lycopolis (Égypte) à l'Académie des Inscriptions et Belles-Lettres
}

\author{
Jean-Luc Fournet, Jean Gascou
}

\section{To cite this version:}

Jean-Luc Fournet, Jean Gascou. Un lot d'archives inédit de Lycopolis (Égypte) à l'Académie des Inscriptions et Belles-Lettres. Comptes-rendus des séances de l'Académie des inscriptions et belleslettres, 2010, 152 (3), pp.1041-1074. hal-01597126

\section{HAL Id: hal-01597126 https://hal.science/hal-01597126}

Submitted on 28 Sep 2017

HAL is a multi-disciplinary open access archive for the deposit and dissemination of scientific research documents, whether they are published or not. The documents may come from teaching and research institutions in France or abroad, or from public or private research centers.
L'archive ouverte pluridisciplinaire HAL, est destinée au dépôt et à la diffusion de documents scientifiques de niveau recherche, publiés ou non, émanant des établissements d'enseignement et de recherche français ou étrangers, des laboratoires publics ou privés. 


\title{
COMMUNICATION
}

\author{
UN LOT D'ARCHIVES INÉDIT DE LYCOPOLIS (ÉGYPTE) \\ À L'ACADÉMIE DES INSCRIPTIONS ET BELLES-LETTRES, \\ PAR M. JEAN-LUC FOURNET ET JEAN GASCOU
}

Pourquoi se fatiguer sur le terrain archéologique alors que les bibliothèques et musées parisiens recèlent tant de trésors documentaires négligés depuis des décennies sinon des siècles ? De fait, à quelques exceptions près comme le fonds démotique ${ }^{1}$ et une pièce homérique sur parchemin (trente-trois vers du chant XVII de l'Odyssée) $)^{2}$, ce n'est que depuis une trentaine d'années que la riche collection de papyrus de l'Académie des inscriptions, constituée au début du $\mathrm{XIX}^{\mathrm{e}}$ siècle et au début du XX $\mathrm{XX}^{\mathrm{e}}$, a commencé à sortir de l'oubli : textes carbonisés du Mendésien, publiés par Sophie $\mathrm{Kambitsis}^{3}$, ceux d'Herculanum, en cours d'étude par Daniel Delattre ${ }^{4}$ et son groupe de travail, viennent enfin les quelque cent vingt papyrus grecs byzantins de Lycopolis (Assiout en MoyenneÉgypte $)^{5}$.

Ces pièces n'ont pas toutes été écrites à Lycopolis. Une minorité substantielle provient des cités méridionales voisines d'Antaiopolis et d'Apollonopolis Parva, mais cela s'explique par des relations

Les papyrus sont cités selon les abréviations de la Checklist of Editions of Greek, Latin, Demotic and Coptic Papyri, Ostraca and Tablets (<http://scriptorium.lib.duke.edu/papyrus/texts/clist.html $>$ ). Les références à Mertens-Pack ${ }^{3}$ renvoient à la base des papyrus littéraires non-chrétiens mise en ligne par le Cédopal (http://promethee.philo.ulg.ac.be/cedopal/index.htm).

1. Étudié par W. Spiegelberg qui l'emporta directement depuis le Caire et, du fait des perturbations que la guerre mondiale provoqua dans les relations scientifiques, ne le rendit à l'Académie qu'en 1930, mais restauré et soigneusement mis sous verres. Seymour de Ricci décrivait le lot au moment de l'achat comme comprenant environ deux cents fragments dont de grandes pièces littéraires. Voir M. Muszynski, Enchoria 6, 1976, p. 19-27.

2. S. de Ricci, «Fragments du chant XVII de l'Odyssée sur un parchemin de Lykôn Polis », Comptes Rendus de l'Académie des Inscriptions et Belles-Lettres 1905, p. 215-217 ; = MertensPack $^{3} 1126$.

3. Voir la préface de son P.Thmouis 1, p. ix (Le papyrus Thmouis 1, colonnes 68-160, Paris, 1983).

4. « Le retour du PHerc.Paris 2 à l'Institut de France : un rouleau épicurien inédit en 279 fragments ", Comptes Rendus de l'Académie des Inscriptions et Belles-Lettres, juillet-octobre 2004, p. 1351-1391 (Philodème de Gadara, Traité sur la calomnie).

5. L'« oubli » est compréhensible en l'occurrence, car les papyrus étaient inventoriés comme des « objets » (les boîtes de fer blanc 193, 194, 195, 197 où les fragments étaient conservés) et moins aisés à repérer. 
d'affaires ou des liens de famille et non pas par des mélanges modernes.

Elles ne peuvent laisser les philologues et les historiens indifférents, car les textes provenant de Lycopolis sont rares, surtout ceux de l'époque tardive. Par ailleurs, nos papyrus se distribuent sur les années 420 à 555 , et surtout le $V^{e}$ siècle, période encore sous-représentée dans notre documentation. Enfin, ces textes, et cela redouble leur intérêt historique, se présentent en archives, en ce sens que leur majorité se regroupe autour de deux ou trois familles, ces familles étant elles-mêmes en relation du fait des alliances matrimoniales ou des transactions.

Les circonstances de leur acquisition ont déjà été exposées par l'un d'entre nous ${ }^{6}$. Il s'agit d'un achat effectué au Caire en février et mars 1905 par le philologue et historien d'art franco-anglais Seymour de Ricci ${ }^{7}$, pour le compte de l'Académie (probablement à l'initiative et avec le concours financier des frères Reinach). Il parla du dossier à plusieurs reprises dans les Comptes Rendus de l'Académie des Inscriptions et Belles-Lettres et dans son « Bulletin papyrologique » de 1921 en des termes montrant qu'il en avait saisi l'importance ${ }^{8}$. Puis ce fut le silence, jusqu'à ce que Paul Lemerle, membre de l'Académie, ne facilite l'accès au dossier, ou plutôt aux innombrables fragments dont il se composait encore en 1987, mis pêle-mêle dans des chemises rangées dans des boîtes métalliques remontant à l'achat (fig. 1). Sous le patronage éclairé de M. Jean Leclant, Secrétaire perpétuel de l'Académie des Inscriptions et Belles-Lettres et de M. Gilbert Dagron, académicien, professeur au Collège de France, ces pièces ont été restaurées au Collège de France en 1997 par Jean-Luc Fournet, remontées, mises sous verres, cotées et numérisées (fig. 2). Précisons que nous n'avons opéré que sur la partie grecque du dossier, alors qu'il comporte de nombreux fragments coptes (surtout des lettres entre particuliers) que nous n'avons ni restaurés ni traités.

6. J. Gascou, « Les papyrus lycopolites de l'Académie des Inscriptions », Atti del XXII Congresso Internazionale di Papirologia. Firenze, 23-29 agosto 1998, I, Florence, 2001, p. 539-547 et pl. XXII.

7. Montefiore Robert Rosso Seymour de Ricci (1881-1942), collectionneur, philologue et historien d'art franco-britannique aux intérêts scientifiques encyclopédiques. Il était très lié aux mécènes et savants frères Reinach, notamment à Théodore, dont on connaît l'intérêt pour la papyrologie.

8. Revue des études grecques 34, 1921, p. 200-201. L'auteur savait de quoi il parlait, car nous avons trouvé dans les boîtes plusieurs copies de papyrus (l'une d'entre elles, celle du $\mathrm{n}^{\circ} 51$, conservant quelques parties disparues). Il est clair aussi que $\mathrm{S}$. de Ricci avait amorcé un travail de mise à plat et de remontage. 


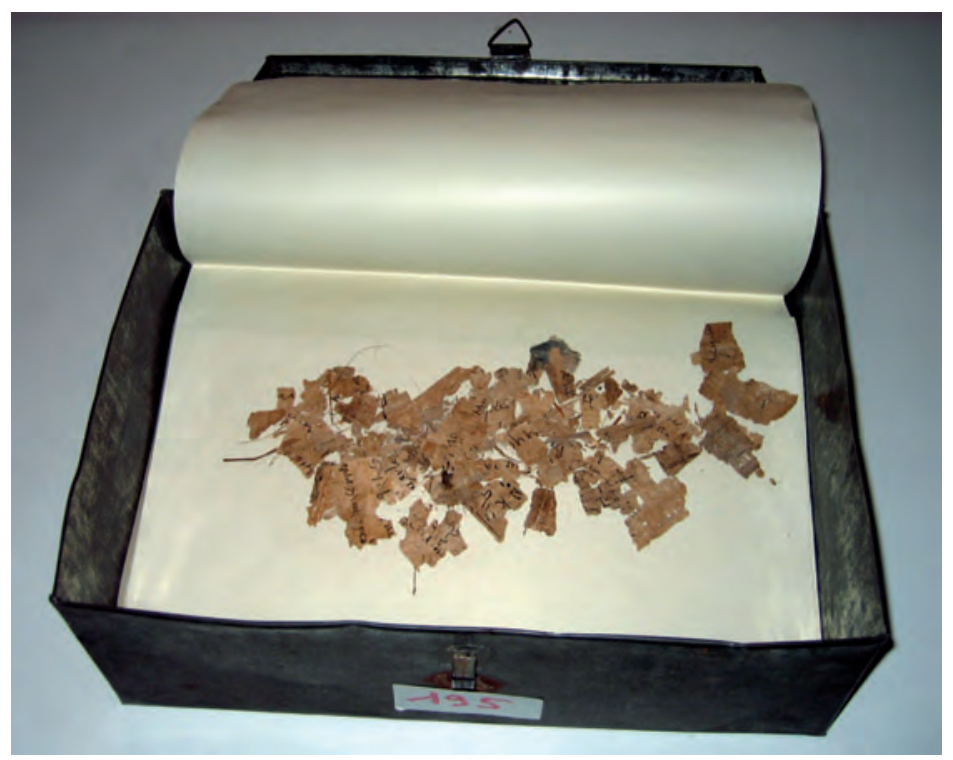

FIG. 1. - Détail du lot avant restauration.

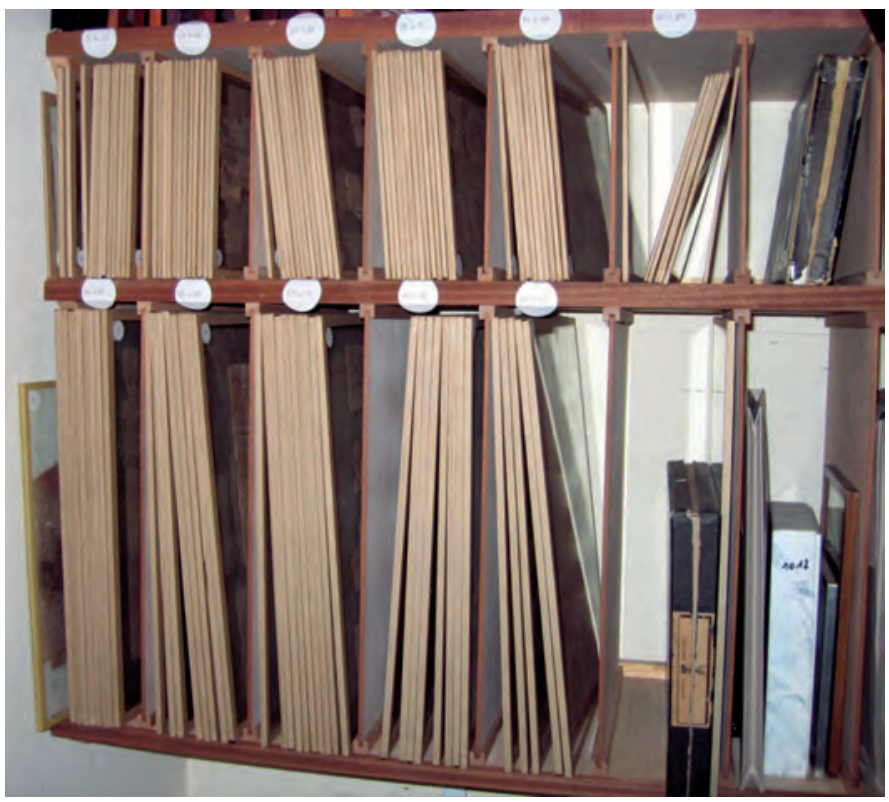

FIG. 2. - Le lot après restauration. 
Peut-on être sûr que l'Académie possède la totalité de la trouvaille originelle ? Elle doit avoir l'essentiel, mais il est possible que certaines pièces aient été distraites avant et après l'achat de Seymour de Ricci. La chose paraît envisageable en raison de sa date (481) pour un rouleau de Princeton qui enregistre une conciliation (dialysis) entre l'évêque Kyros de Lycopolis et ses frères, d'une part, et un de ses diacres, de l'autre, en litige sur de petites affaires'. D'après un raccord fait par J.-L. Fournet (sur lequel il reviendra) entre un fragment d'Aristophane du lot de l'Académie et des fragments de la Bodléienne, d'autres pièces se trouvent à Oxford. Appartiennent presque certainement au dossier un fragment de Vienne publié en $2004^{10}$ et un contrat de prêt fragmentaire daté de 582 conservé à Yale ${ }^{11}$.

Toutefois, en 1932 (ou 1942 ?), Seymour de Ricci donna à la Bibliothèque nationale un important ensemble de papyrus et de parchemins, dont des documents, « rapportés d'une mission en Égypte en 1905 », et aujourd'hui conservés au département des manuscrits orientaux de la BnF sous la cote BN Copte 166. J.-L. Fournet a examiné récemment ce fonds et a retrouvé deux textes de Lycopolis qui auraient pourtant dû rester à l'Académie. Comme on voit, le dossier de Lycopolis peut encore s'accroître et cela nous fait moins regretter notre retard.

Sa publication en un volume unique, mobilisant le concours de savants étrangers, Fabian Reiter et Fritz Mitthof, est notre projet scientifique commun le plus urgent, encore que certains textes soient déjà publiés ${ }^{12}$.

Nous souhaitons ici donner une idée des genres qu'ils illustrent, en insistant sur quelques pièces significatives.

9. SB III 7033. On trouve aussi dans la réédition partielle P.Princ. II 82 diverses corrections

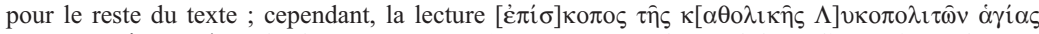
$\tau$ นิ $\Theta \varepsilon \circ \hat{v}[\dot{\varepsilon} \kappa \kappa \lambda \eta \sigma i \alpha \varsigma]$ des 1. 4-5 est peu conforme au protocole usuel des églises épiscopales. On

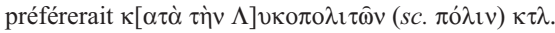

10. CPR XIX 8 de 481 (corrections de D. Hagedorn, « Bemerkungen zu Urkunden », Zeitschrift für Papyrologie und Epigraphik 150, 2004, p. 155), peut-être adressé par Fl. Patrikios (personnage attesté dans les papyrus de l'Académie des Inscriptions et Belles-Lettres), détaché au bureau militaire provincial.

11. PCtYBR inv. 4478 (A. Benaissa, "Four Byzantine Papyri with Notarial Subscriptions », Zeitschrift für Papyrologie und Epigraphik 160, 2007, p. 222-224 avec pl.).

12. Les gloses de P.Acad. inv. 3/4 ; les textes médicaux conservés par P.Acad. inv. 4-6 ; la pétition de P.Acad. inv. 18/2 ; le transfert de bail P.Acad. inv. 51 (SB XXVI 16532) ; le procès-verbal d'audience P.Acad. inv. 56/1-2 + 57/1, la quittance de loyer inv. 55 (A. Papaconstantinou, Le culte des saints en Égypte des Byzantins aux Abbassides, Paris, 2001, p. 380). On reviendra plus loin sur certains de ces papyrus. 


\section{Le dossier littéraire et son intérêt culturel}

Sur les quelque 120 pièces de ce lot, 23 ne sont pas des documents mais des textes littéraires ou paralittéraires - sans compter un dessin assez grossier sur le verso d'un compte. Si on laisse de côté les fragments trop abîmés pour que leur contenu soit identifiable, cet ensemble contient 5 textes littéraires et 9 textes paralittéraires.

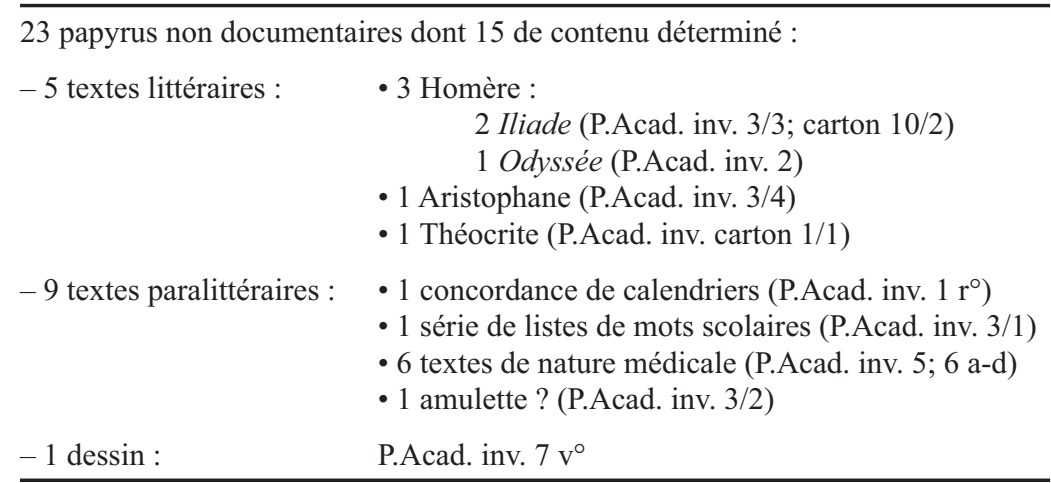

23 papyrus sur 120 , voilà qui constitue une proportion assez forte (supérieure à celle que l'on constate en général entre papyrus littéraires et documentaires, de l'ordre de 1/10). Mais il faut tenir compte du mauvais état de conservation de ces textes, qui se réduisent souvent à de petits fragments. Cet état contraste avec celui des documents qui, après remontage, sont beaucoup plus complets. Cette constatation oblige à s'interroger sur une possible contamination de ce lot : quelques bribes (notamment littéraires) de provenances diverses auraient pu en effet se glisser dans l'ensemble lycopolitain à la faveur de manipulations modernes. L'hypothèse pourrait plus précisément s'appuyer sur un papyrus d'Aristophane, raccordé par J.-L. Fournet à trois fragments appartenant à la Bodléienne (fig. 7-8) ${ }^{13}$. Or ces derniers avaient été publiés en 1905 par B.P. Grenfell et A.S. Hunt qui les avaient achetés « en décembre 1904 au Caire, sélectionnés dans une boîte pleine de papyrus byzantins d'Ashmunen » (l'ancienne Hermopolis) ${ }^{14}$. Le fragment de l'Académie comme

13. Bodl. Ms. Gr. class. f. 72 (P), édité par B.P. Grenfell et A.S. Hunt, " Some Classical Fragments from Hermopolis ", Mélanges Nicole, I, Genève, 1905, p. 212-217.

14. B.P. Grenfell et A.S. Hunt, l. c., p. 212. C'est à ce titre qu'ils sont compris dans la liste des textes littéraires originaires d'Hermopolis dressée par P. van Minnen et K.A. Worp, "The Greek and Latin Literary Texts from Hermopolis », Greek Roman and Byzantine Studies 34, 1993, p. $151-186$. 
ceux de la Bodléienne ont été acquis à peu près au même moment : décembre 1904 (Grenfell et Hunt), début 1905 (de Ricci). Il est vraisemblable que les achats ont eu lieu chez le même antiquaire, ce qui a pu donner lieu à des mélanges, sans qu'on puisse établir dans quel sens se produisit la contamination. Voilà qui pourrait jeter un doute sur la provenance de toutes les pièces littéraires de notre lot. Leur datation semble cependant favoriser une appartenance au reste des archives : l'amplitude chronologique de ces textes ( $\mathrm{du} \mathrm{IV}^{\mathrm{e}}$ - peut-être fin $\mathrm{III}^{\mathrm{e}}-\mathrm{au} \mathrm{VI} \mathrm{I}^{\mathrm{e}}$ siècle, avec un noyau dur du $\mathrm{V}^{\mathrm{e}}$, siècle pourtant mal représenté en papyrus littéraires comme documentaires) correspond bien à ce que nous savons du dossier documentaire, s'échelonnant de 420 à 555. Qu'il y ait des papyrus du IV e siècle, soit de quelques décennies, voire peut-être d'un siècle, antérieurs aux premiers documents, n'a d'ailleurs rien pour étonner : les exemplaires de textes littéraires pouvaient se transmettre d'une génération à l'autre jusqu'à ce qu'ils deviennent inutilisables et soient mis au rebut ; cela explique que, lorsqu'ils se rattachent à des archives, les textes littéraires ont souvent un " centre de gravité » chronologique antérieur aux documents. On notera d'ailleurs que les papyrus littéraires les plus anciens de notre lot contiennent les poèmes homériques, l'abc de la culture littéraire qui a traversé toutes les modes culturelles et qui se transmettait donc jusqu'à usure totale - l'un d'entre eux a même été réutilisé dans la reliure en cuir d'un livre ${ }^{15}$.

Nous venons de parler de connexion entre papyrus littéraires et documentaires. C'est précisément là un des intérêts de cet ensemble littéraire, quelque fragmentaire qu'il soit. Dans bien des cas, les papyrus littéraires, parce que trouvés clandestinement, sont en effet dénués de toute information sur leur origine. Quant aux ensembles cohérents dont on connaît le milieu de constitution - ce que les papyrologues dénomment " archives »-, ils ne contiennent la plupart du temps que des documents. C'est pourtant la conjonction des deux qui permet seule de remettre précisément les livres antiques dans le contexte socio-culturel de leurs lecteurs ${ }^{16}$. Ce lot

15. P.Acad. inv. 2 (Homère, Od. XVII 134-136; 200-208; 227-234; 299-308; 323-332). Cf. ci-après.

16. Les quelques cas de textes littéraires qui puissent être replacés dans un contexte documentaire ont été étudiés par W. Clarysse, "Literary Papyri in Documentary "Archives" ", dans Egypt and the Hellenistic World, Studia Hellenistica 27, Louvain, 1983, p. 43-61. « (...) It is often very difficult to connect literary texts with an archive because we usually cannot base ourselves on internal evidence of the texts and, secondly, (...) in many cases a person's papers are preserved but not his library (or vice versa)» (p. 61). 


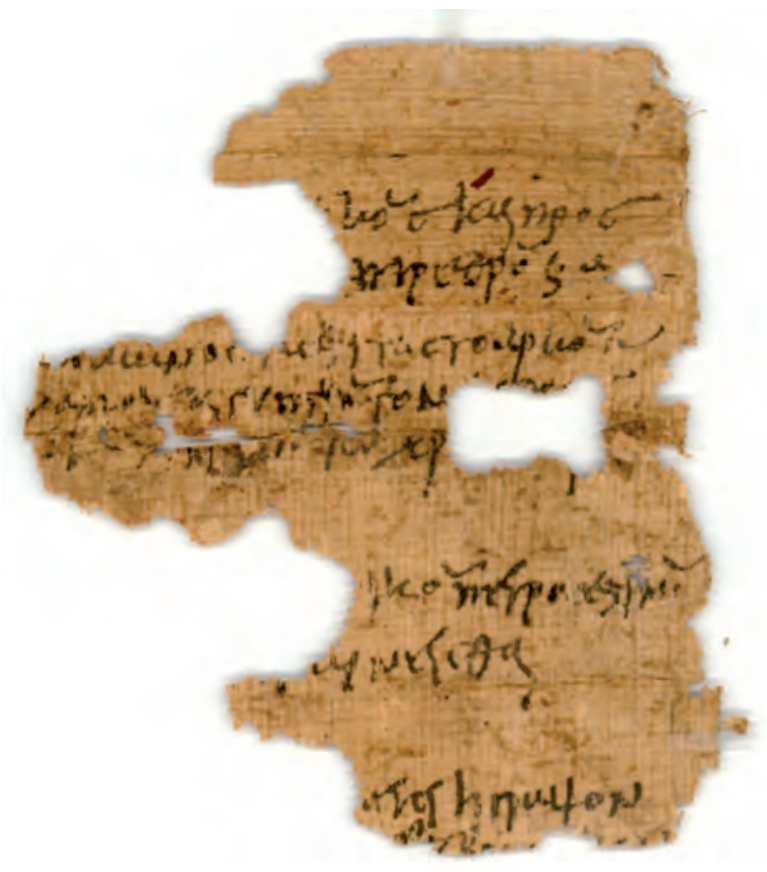

FIG. 3. - Texte à contenu médical (P.Acad. inv. 6/1).

de Lycopolis présente donc la particularité de conjuguer ces deux types d'écrit, mais cet intérêt est quelque peu affaibli par l'impossibilité où nous nous trouvons de mettre ces livres en rapport avec une des personnalités des archives. Ce constat est à nuancer avec les papyrus médicaux dont le nombre incite peut-être à supposer l'existence d'un médecin, d'un apothicaire - ou en tout cas d'un individu intéressé par la médecine - parmi les acteurs de ces archives. Il n'a pu pour l'instant être identifié avec les personnages nommés dans les documents ; il est néanmoins probable qu'un de ces textes, le P.Acad. inv. 6/1 (fig. 3), un ensemble de recettes ou une lettre à contenu médical, soit de la même main que les P.Acad. inv. 1 (fig. 9-10) et 49 (fig. 4), deux brouillons de pétitions adressées au préfet du prétoire - mais ces textes, incomplets, n'ont pas livré le nom de leur auteur.

Malgré ces réserves, ce petit groupe littéraire et paralittéraire n'est pas sans intérêt philologique et culturel comme nous allons le voir. 


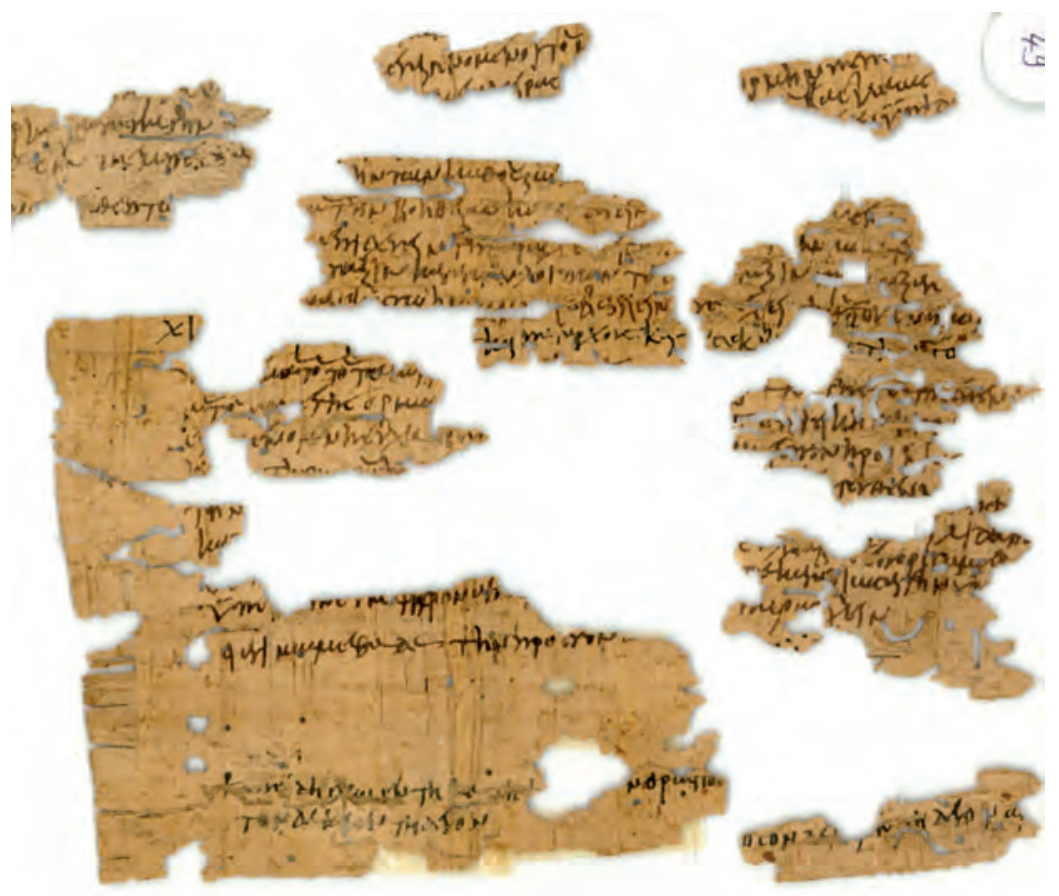

FIG. 4. - Pétition (P.Acad. inv. 49, détail).

\section{LES PAPYRUS HOMÉRIQUES}

Nous passerons vite sur deux minuscules fragments de codex sur papyrus, P.Acad. inv. $3 / 3$ et carton 10/2, donnant l'Iliade X 347-353 et XVI 276-290, datant respectivement du $\mathrm{III}^{\mathrm{e}} / \mathrm{IV}^{\mathrm{e}}$ et $\mathrm{IV}^{\mathrm{e}} / \mathrm{V}^{\mathrm{e}}$ siècle. Plus intéressant est le P.Acad. inv. 2 (fig. 5), restes de trois folios d'un codex de parchemin conservant une partie du chant XVII de l'Odyssée (134-136, 200-208, 227-234, 299-308, 323-332). Les traces de cuir que l'on relève sur la surface du parchemin montrent que ces feuillets ont été remployés dans une reliure en cuir pour un ouvrage postérieur au IV $\mathrm{V}^{\mathrm{e}}$ siècle, date de la copie de cet exemplaire. Comme on l'a déjà signalé, deux de ces folios ont été édités par de Ricci ${ }^{17}$; nous avons pu identifier un troisième fragment et, en reprenant l'étude de l'ensemble, en dégager la cohérence

17. S. de Ricci « Fragments du chant XVII de l'Odyssée sur un parchemin de Lykôn Polis », Comptes Rendus de l'Académie des Inscriptions et Belles-Lettres 1905, p. 215-217 ; = MertensPack $^{3} 1126$. 

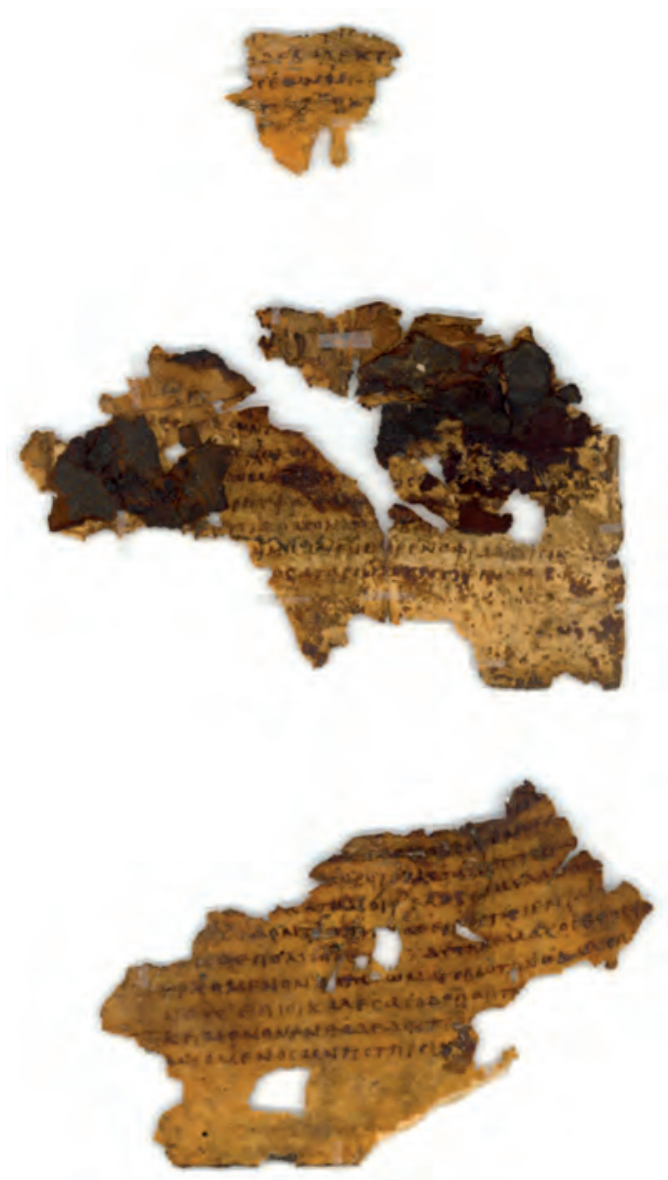

FIG. 5. - Homère, Odyssée (P.Acad. inv. 2).

codicologique : nous avons affaire au reste d'un cahier (un quinion au minimum) d'un codex dont les dimensions devaient être env. H 18 x L 12,5 cm (format précisément répertorié pour des éditions $\mathrm{du} \mathrm{III} / \mathrm{IV}^{\mathrm{e}} \mathrm{s} .{ }^{18}$ ) et dont chaque page devait contenir 24 ou 25 lignes.

L'intérêt de cet exemplaire tient moins à son texte (on ne relève aucune variante) qu'aux signes diacritiques dont il est généreusement pourvu : apostrophes et trémas de première main, accents, esprits

18. Il entre dans les aberrants 1 du groupe 9 d'E. Turner, The Typology of the Early Codex, Philadelphia, 1977, p. 22. Turner répertorie notre codex sous le $\mathrm{n}^{\circ} 194$ de sa table 16 . Nous apportons ici des corrections et des compléments. 


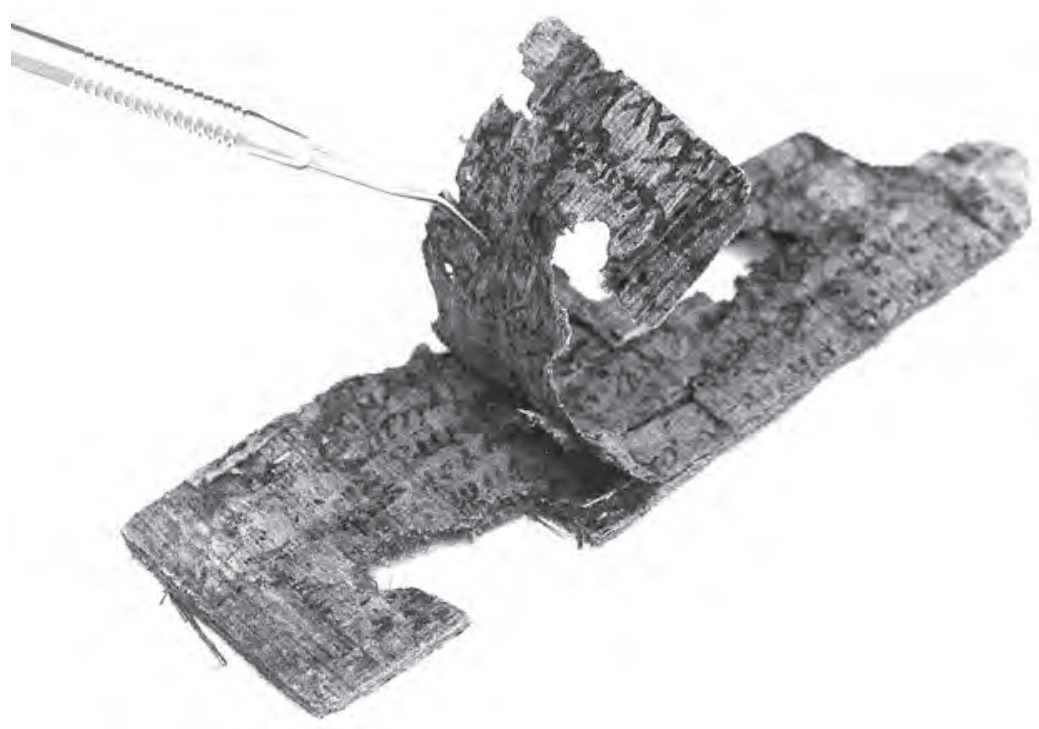

FIG. 6. - Extraction du papyrus de Théocrite du carton 1.

rudes et ponctuation (haute et peut-être basse) ajoutés postérieurement. On notera l'absence d'esprit doux, phénomène fréquent dans les papyrus, permettant de déduire que les Anciens ne voyaient pas l'utilité de marquer l'absence d'aspiration sauf en cas d'amphibolie. Quant aux accents, qui tiennent du système byzantin et non plus alexandrin, on relève certaines particularités graphiques : le circonflexe tend à empiéter sur la lettre suivante alors que le grave se déporte sur la lettre, voire la syllabe précédentes, selon une tendance fréquente dans les papyrus de cette époque ${ }^{19}$.

\section{LE PAPYRUS DE THÉOCRITE (P. Acad. inv. carton 1/1)}

Ce fragment a été récupéré dans un des « cartons » rectangulaires, de confection ancienne, que contenait notre lot et dont la destination n'est pas encore claire (fig. 6). Il appartient au folio d'un codex de papyrus dont les dimensions devaient avoisiner les H 30 x L $19 \mathrm{~cm}$ et dont chaque page contenait au moins 46 lignes. Son écriture, une capitale penchée, invite à le dater du début du $\mathrm{VI}^{\mathrm{e}}$ siècle ${ }^{20}$. Il livre

19. Cf. P.Aphrod.Lit., p. 26-29.

20. Comparer G. Cavallo et H. Maehler, Greek Bookhands, $\mathrm{n}^{\circ} 27 \mathrm{~b}$ (première moitié du VI $\mathrm{s}$.). 
deux variantes dont une nouvelle mais peu intéressante ${ }^{21}$. Cependant, comme il contient sur une face Idylles VII 112-120 et sur l'autre Idylles III 1-8, il apporte un témoignage supplémentaire de la succession Idylles VII-III, déjà donnée par trois autres papyrus des $\mathrm{II}^{\mathrm{e}}$ et $\mathrm{VI}^{\mathrm{e}}$ siècle $^{22}$ ainsi que par le ms K (Ambrosianus 886, XIII ${ }^{\mathrm{e}} \mathrm{s}$.) et six manuscrits de la recension dite laurentienne ${ }^{23}$. C'est d'ailleurs le seul ordre connu par les papyrus pour les Idylles VII et III ${ }^{24}$.

LE PAPYRUS D'ARISTOPHANE (P.Acad. 3/4 + P.Bodl. Ms. Gr. class.

f. $72(P)=$ Mertens-Pack ${ }^{3}$ 141) .

C'est de loin le papyrus littéraire le plus intéressant. On en trouvera l'édition en Appendice 1.

Cet ensemble de papyrus littéraires appelle quelques remarques générales. Il se distingue par son adéquation avec les tendances culturelles qui lui sont contemporaines - mise à part la surreprésentation d'Homère, un phénomène permanent qui correspond au rôle d'exception de cet auteur fondateur. La présence dans ces archives d'une édition d'Aristophane de la fin du IV ou du début du V $V^{\mathrm{e}}$ siècle reflète le regain d'intérêt pour cet auteur qui, dans les statistiques papyrologiques, après une période d'indifférence, supplante son rival Ménandre, précisément entre le $\mathrm{IV}^{\mathrm{e}}$ et $\mathrm{V}^{\mathrm{e}}$ siècle ${ }^{25}$. Cette redécouverte

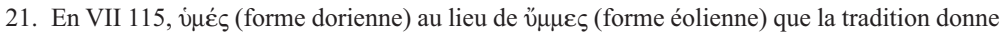

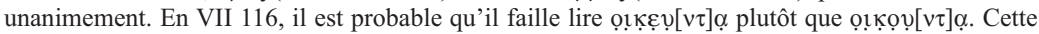
dernière est de toute façon une correction de Hecker, adoptée par les éditeurs modernes. Les mss

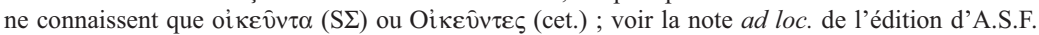
Gow.

22. P.Oxy. 2064 (éd. A.S. Hunt et J. Johnson, Two Theocritus Papyri, Londres, 1930) + L 3548 (= Mertens-Pack ${ }^{3}$ 1489), II s. ; P.Berol. 21182 (éd. H. Maehler, " Griechische Literarische Papyri », Zeitschrift für Papyrologie und Epigraphik 4, 1969, p. 114-116 = Berliner Klassikertexte IX 85 ; Mertens-Pack $\left.{ }^{3} 1489.4\right), \mathrm{VI}^{\mathrm{e}}$ s. ; et P.Oxy. LXIV 4430 (= Mertens-Pack $\left.{ }^{3} 1489.41\right), \mathrm{II}^{\mathrm{e}} \mathrm{s}$.

23. P (Laurentianus XXXII.37, XIII $-\mathrm{XIV}^{\mathrm{e}}$ s.), Q (Parisinus gr. 2884, 1299), T (Vaticanus gr. 38, 1322), V (Vaticanus gr. 1824, XVe s.), W (Laurentianus Conv. Soppr. 15, XIVe s.) et Tr (Parisinus gr. 2832, $\mathrm{XIV}^{\mathrm{e}} \mathrm{s}$.).

24. Sur ce problème d'ordre des Idylles, cf., entre autres, l'éd. de Théocrite par A.S.F. Gow, Cambridge 1965, p. LXVI-LXIX ; J. Irigoin, « Les Bucoliques de Théocrite. La composition du receuil », Quaderni urbinati di cultura classica 19, 1975, p. 27-44 ; K. Gutzwiller, " The Evidence for the Theocritean Books » dans M.A. Harder, R.F. Regtuit, G.C. Wakker (éd.), Theocritus, Hellenistica Groningana II, Groningen 1995, p. 119-148 ; A. Blanchard, « L'architecture secrète des Bucoliques de Théocrite », Bulletin de l'Association Guillaume-Budé 2006/1, p. 100-118, repris dans Id., Dans l'ouvroir du poète, Paris, 2008, p. 117-131.

25. Pour les chiffres, que l'on actualisera avec ceux de la base Mertens-Pack ${ }^{3}$ du Cédopal, cf. P. Mertens, "Les papyrus d'Aristophane : actualisation des données bibliologiques et

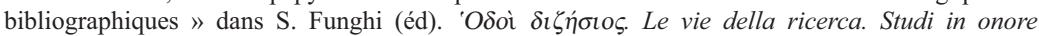
di Francesco Adorno, Firenze, 1996, p. 335-344 et M. Manfredi dans P.Bingen, p. 97-98. Pour 
d'Aristophane à l'époque protobyzantine se traduit par la diffusion d'éditions pourvues de scolies marginales comme la nôtre, proportionnellement si nombreuses qu'elles constituent pour un auteur un des ensembles de codex annotés le plus fourni observable en papyrologie littéraire ${ }^{26}$.

Le papyrus de Théocrite est aussi un reflet fidèle des modes littéraires de l'époque : sur les vingt-trois papyrus de Théocrite actuellement connus couvrant le millénaire papyrologique, il est le sixième postérieur au $\mathrm{IV}^{\mathrm{e}}$ siècle $^{27}$, ce qui, compte tenu de l'effondrement de la production libraire après ce siècle, est le signe d'un regain d'intérêt pour Théocrite. Ce regain correspond à celui que l'on observe plus généralement pour la poésie en Egypte à cette même période ; il se vérifie par les références que les poètes tardifs font à Théocrite, notamment Nonnos de Panopolis ( $V^{\mathrm{e}} \mathrm{s}$.) ; il est enfin à mettre en rapport avec l'activité philologique dont cet auteur a fait l'objet entre le $\mathrm{IV}^{\mathrm{e}}$ (avec Munatius) et le $\mathrm{VI}^{\mathrm{e}}$ siècle (avec Thééthète le Scholastique et Érathosthène $)^{28}$.

Une absence se fait en revanche remarquer dans notre ensemble de papyrus littéraires, celle des textes chrétiens, surprenante, mais difficile à expliquer actuellement.

\section{PAPYRUS PARALITTÉRAIRES}

À côté de ces bribes de livres, notre lot comprend une dizaine de papyrus paralittéraires - textes qui se situent à la frange de la littérature sans en être, qu'on pourrait appeler, en usant d'un oxymore, des « documents littéraires ».

leur interprétation, voir A. Blanchard, «Destins de Ménandre », Ktèma 22, 1997, p. 218-224 et F. Montana, loc. cit. à la note suivante.

26. Cf. K. McNamee, Marginalia and Commentaries in Greek Literary Papyri, diss. Duke Univ. 1997, p. 177, qui remarque que les papyrus annotés d'Aristophane forment « a larger group of texts than any so far considered for a single author » (cf. en dernier lieu K. McNamee, « Annotations in Greek and Latin Texts from Egypt », American Studies in Papyrology 45, 2008, notamment p. 57). On compte actuellement 18 papyrus annotés de cet auteur (sans compter les hypomnêmata et les lexiques), récemment rassemblés dans G. Bastianini, M. Haslam, H. Maehler, F. Montanari et C. Römer (éd.), Commentaria et Lexica Graeca in Papyris Reperta, Pars I, vol. 1, fasc. 4, Munich, 2006, p. 13-240. Cette édition est pourvue d'une synthèse due à F. Montana (p. 3-12). Voir aussi H. Maehler, « Die Scholien der Papyri in ihrem Verhältnis zu den Scholiencorpora der Handschriften », La Philologie grecque à l'époque hellénistique et romaine, Entretiens de la Fondations Hardt, XL, Genève, 1994, p. 124-127.

27. Outre le nôtre : P.Oxy. XIII 1618 (= Mertens-Pack ${ }^{3}$ 1490), V s. ; Mertens-Pack 1487 , $\mathrm{V}^{\mathrm{e}} / \mathrm{VI}^{\mathrm{e}}$ s. ; Mertens-Pack ${ }^{3}$ 1488, Ve/VI ${ }^{\mathrm{e}}$ s. ; Berliner Klassikertexte IX 85 (= Mertens-Pack ${ }^{3}$ 1489.4), $\mathrm{VI}^{\mathrm{e}} \mathrm{s}$. ; Berliner Klassikertexte VI, p. 55 (= Mertens-Pack $\left.{ }^{3} 1491\right), \mathrm{VI}^{\mathrm{e}} / \mathrm{VII}^{\mathrm{e}} \mathrm{s}$.

28. Cf. A.S.F. Gow dans son édition, p. LXXXIII-LXXXIV, suivant Ahrens. 
Au moins deux d'entre eux sont scolaires ${ }^{29}$ :

- Le P.Acad. inv. carton 10/1 conserve les restes d'une table de conjugaison du verbe ßoó $\omega$, verbe modèle, depuis Denys le Thrace $\left(\mathrm{II}^{\mathrm{e}} / \mathrm{I}^{\mathrm{er}} \mathrm{av}\right.$.), des verbes contractes en $-\alpha \omega^{30}$. La présentation de la matière verbale, telle qu'elle apparaît dans les quelques lignes de ce fragment, donne à penser que notre texte suivait l'ordonnancement des Canons élémentaires de la conjugaison verbale du grammairien Théodose d'Alexandrie (IV/ $/ \mathrm{v}^{\mathrm{e}} \mathrm{s}$.) ${ }^{31}$, qui est aussi celui de la majorité des papyrus de conjugaison ${ }^{32}$.

- Le P.Acad. inv. 3/1 est un feuillet donnant deux listes de mots, dont le dénominateur commun et la finalité ne sont pas toujours clairs, mais qui doivent être replacées dans un contexte scolaire - ce que confirme sa mauvaise orthographe.

Les autres textes sont de nature diverse. Ainsi le P.Acad. inv. $1 \mathrm{r}^{\circ}$, dont on trouvera l'édition en Appendice 2, livre une concordance entre les calendriers égyptien, julien et macédonien. Contrairement à la plupart des autres concordances, ce texte n'est pas de nature scolaire. À la personne qui l'a copié on doit aussi le texte du verso (fig. 10) et d'autres documents du dossier (fig. 3-4) : il ne peut s'agir d'un élève. La fonction de ce texte est en fait purement pratique : il répond au besoin de pouvoir confronter - même de façon approximative puisque les mois des trois systèmes ne commencent pas exactement le même jour - le calendrier égyptien avec les deux principaux calendriers utilisés à l'époque dans l'Empire. Le calendrier

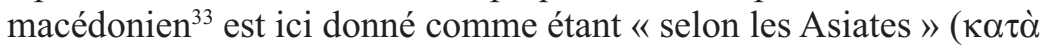
'Aøı avoús), autrement dit les ressortissants du diocèse d'Asie ; c'est aussi celui qui était d'usage préférentiel dans le diocèse d'Orient au $V^{\mathrm{e}}$ siècle. Le calendrier julien, quant à lui, était utilisé dans la capitale de l'Empire, Constantinople. Aussi, par exemple, lorsque l'évêque alexandrin Athanase ( $\mathrm{IV}^{\mathrm{e}} \mathrm{s}$.) cherche à établir une correspondance pour un mois, est-ce précisément entre les trois calendriers retenus par notre concordance qu'il le fait ${ }^{34}$.

29. Sans compter l'exercice d'écriture visible dans la marge inférieure du recto et sur le verso de P.Acad. inv. 52, une lettre dont quelqu'un s'est entraîné à recopier certains passages.

30. Cf. P.Aphrod.Lit., p. 221

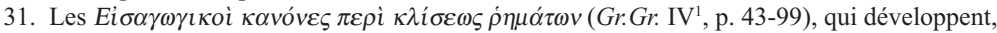
avec commentaire, toute la conjugaison de $\tau \dot{\pi} \pi \tau \omega$, et qui sont résumés sous la forme d'une table, donnée dans l'appendice IV au Gr.Gr. I'

32. Cf. P.Aphrod.Lit., p. 226.

33. Cf. V. Grumel, La Chronologie, Traité d'études byzantines, I, Paris, 1958, p. 168-169.

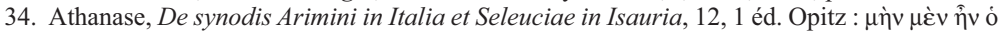

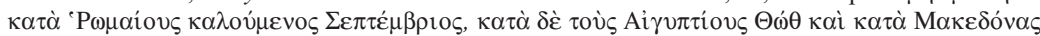

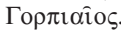


Ce n'est pas un hasard si cette concordance est de la même main que les brouillons de pétitions conservés sur le verso (fig. 10) ou sur le P.Acad. inv. 49 (fig. 4), deux textes ${ }^{35}$ adressés au préfet du prétoire d'Orient ${ }^{36}$. Dans la requête qu'il s'apprêtait à remettre à ce haut fonctionnaire, dont le siège était à Constantinople, ou dans les démarches qu'il allait entreprendre à cette occasion, l'auteur du P.Acad. inv. 1 a dû éprouver le besoin de donner des équivalences de dates qui soient compréhensibles par des non-Égyptiens.

Toujours dans le domaine du paralittéraire, signalons enfin un groupe singulier de papyrus de nature médicale, publié ailleurs par J.-L. Fournet ${ }^{37}$, qui, comme on l'a dit, pourrait avoir appartenu à un médecin ou apothicaire, sans doute l'auteur des pétitions conservées par les P.Acad. inv. $1 \mathrm{v}^{\circ}$ et 49 . L'hypothèse d'un apothicaire pourrait s'appuyer sur un des papyrus de ce petit ensemble, le P.Acad. inv. 5, probablement une liste d'ingrédients, qui est

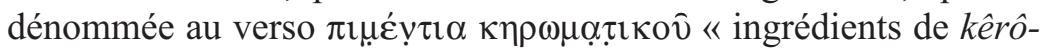
matikos $\|^{38}$, ce qui permet d'y voir une liste de produits destinés à un masseur ou un osthéopathe ${ }^{39}$ et que notre apothicaire aurait été chargé de préparer. Ce n'est là qu'une hypothèse, fragile, mais qui expliquerait les autres recettes médicales du lot, toutes de mains différentes, qui auraient été autant d'ordonnances que notre homme aurait préparées soit pour le médecin soit pour le patient : ainsi

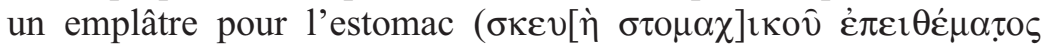

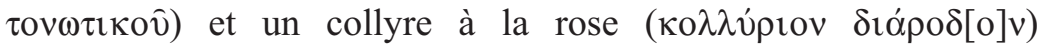
(P.Acad. inv. 4$)^{40}$; une recette contre l'entérocèle ou l'hydrocèle

35. Qui n'en forment peut-être qu'un ou qui sont deux versions de la même requête. Leur incomplétude et l'état très fragmentaire du P.Acad. inv. 49 ne permettent pas de se faire une idée très précise du texte.

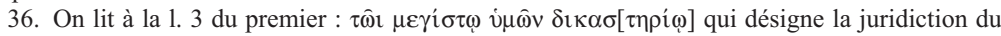
préfet du prétoire. Cf. D. Feissel, « L'ordonnance du préfet Dionysios inscrite à Mylasa en Carie (1 ${ }^{\text {er }}$ août 480) », Travaux et Mémoires 12, 1994, p. 264.

37. J.-L. Fournet, «Un papyrus médical de l'Académie des Inscriptions et Belles-Lettres », Travaux et Mémoires 12, 1994, p. 309-322 (édition du P.Acad. inv. 4) ; " La bibliothèque d'un médecin ou d'un apothicaire de Lycopolis ? » dans I. Andorlini (éd.), Testi medici su papiro. Atti del Seminario di studio (Firenze, 3-4 giugno 2002), Florence, 2004, p. 175-197 (édition des P.Acad. inv. $4 ; 5 ; 6 / 1-4)$.

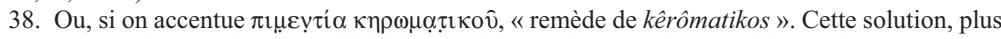
contournée, est discutée dans « La bibliothèque d'un médecin ou d'un apothicaire de Lycopolis? », op. cit., p. 190-191.

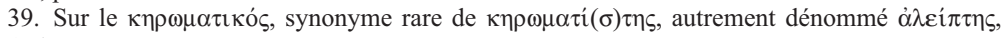
cf. ibid.

40. J.-L. Fournet, «Un papyrus médical de l'Académie des Inscriptions et Belles-Lettres », Travaux et Mémoires 12, 1994, p. 309-322 et « La bibliothèque d'un médecin ou d'un apothicaire de Lycopolis? », op. cit., p. 185-187. 
(P.Acad. inv. 6/4) ;1 $^{4}$ une recette ou une liste d'ingrédients (P.Acad. inv. $6 / 2)^{42}$; une série de recettes ou une lettre à contenu médical (P.Acad. inv. 6/1 $)^{43}$. Enfin, on relèvera la présence d'une étiquette de médicament contre la goutte (P.Acad. inv. 6/3) ${ }^{44}$.

Par les rapports qu'ils laissent entrevoir avec lui, ces textes paralittéraires nous introduisent au pan documentaire de ce lot.

\section{Les textes documentaires et leur intérêt historique}

\section{LES LETTRES}

On compte dix-huit lettres ${ }^{45}$, de sens souvent peu évident du fait du tour elliptique et allusif de l'épistolographie sur papyrus en général et de leur état presque toujours fragmentaire - leur format et de leur mode d'archivage, ont entraîné à plusieurs reprises la perte d'une moitié de feuillet. On notera - ce qui n'est pas indifférent à la connaissance des modes d'écriture de l'époque - qu'elles sont toutes écrites transfibralement, sur des coupons normalement rectangulaires dont le petit côté est vertical ${ }^{46}$. Plusieurs d'entre elles sont adressées à un certain Biktôr, diœcète de la domus divina du nome Antaiopolite, soit intendant des domaines impériaux ${ }^{47}$. Faute de pouvoir entrer ici dans la discussion des problèmes qu'elles soulèvent, on se contentera de signaler l'occurrence de toponymes nouveau ${ }^{48}$, de mots rares et surtout une allusion aux Blemmyes, peut-être motivée par les raids que ces nomades nubiens ont menés en Thébaïde durant le $\mathrm{V}^{\mathrm{e}}$ siècle $^{49}$.

41. J.-L. Fournet, «La bibliothèque d'un médecin ou d'un apothicaire de Lycopolis? », op. cit., p. 177-179. D'autres hypothèses de maladies sont formulées dans l'introduction à l'édition, mais elles sont moins vraisemblables.

42. J.-L. Fournet, « La bibliothèque d'un médecin ou d'un apothicaire de Lycopolis ? », op. cit., p. $179-180$

43. J.-L. Fournet, « La bibliothèque d'un médecin ou d'un apothicaire de Lycopolis ? », op. cit., p. 181-185. Ce texte pourrait être de la main de notre « apothicaire » si on pose l'identité du possesseur de cet ensemble de textes médicaux avec le rédacteur des pétitions.

44. J.-L. Fournet, « La bibliothèque d'un médecin ou d'un apothicaire de Lycopolis ? », op. cit., p. $176-177$.

45. P.Acad. inv. $8 ; 9 ; 10 ; 11 ; 12 ; 13 / 1 ; 13 / 2 ; 14 / 1+20 / 3 ; 14 / 2 ; 15 / 1 ; 15 / 2 ; 16 / 1 ; 16 / 2$; $17 ; 19 ; 52 ; 53 ; 54$.

46. Cf. J.-L. Fournet, « Disposition et réalisation graphique des lettres et des pétitions protobyzantines : pour une paléographie "signifiante" des papyrus documentaires ", Proceedings of the 24th International Congress of Papyrology, Helsinki, 2007, p. 354-358.

47. P.Acad. inv. $8 ; 14 / 2 ; 15 / 1 ; 15 / 2 ; 16 / 1 ; 17 ; 19$.

48. Фvó $\mu \omega t \varsigma$ en P.Acad. inv. $8,1$.

49. P.Acad. inv. 9, 10 . 


\section{LES DOCUMENTS PUBLICS}

\section{Les pétitions}

Le dossier compte au moins trois pétitions sous forme de brouillon. On a déjà évoqué plus haut deux d'entre elles, adressées au préfet du prétoire, peut-être écrites par 1'《 apothicaire » (P.Acad. inv. 1 $\mathrm{v}^{\circ}$ [fig. 10] et 49 [fig. 4]). Elles semblent faire suite à une première procédure engagée au niveau local (sans doute à Alexandrie d'après P.Acad. inv. $\left.1 \mathrm{v}^{\circ}, 8\right)$; le plaignant, non satisfait, s'adresse maintenant en appel au tribunal du préfet du prétoire, ce qui implique de sa part un voyage à Constantinople. L'affaire paraît être allée jusqu'au plus haut sommet de l'Empire puisque P.Acad. inv. 49, fr. H, 3, mentionne des rescrits (syllabas) que le pétitionnaire a obtenus de l'empereur.

S'ajoute à ces deux textes P.Acad. inv. 18/2, brouillon conservant le préambule d'une pétition adressée sans doute à un duc, aux accents très encomiastiques comme c'était alors d'usage : l'auteur y développe une métaphore où il compare l'état de la province à une mer et son gouverneur à un port protecteur ou à un pilote, évoquant les tourments du passé et le calme de la situation présente dû à la bonne administration du duc $\mathrm{c}^{50}$.

\section{Les procès-verbaux de procès gréco-latins}

Quatre documents entrent à ce jour dans cette catégorie. Tous très fragmentaires, ils enrichissent une série de textes contemporains illustrée par quelque cinquante papyrus. La mieux conservée de nos pièces (P.Acad. inv. 56/1-2 + 57/1) se rapporte à une audience du gouverneur militaire de la province de Thébaïde, qui est un personnage historique, Flavius Aspar Nomus Candidianus Caesarius, déjà connu par une inscription du Couvent Blanc, des écrits de l'archimandrite panopolitain Chénouthé et une chronique copte situant son gouvernorat autour de $451^{51}$.

50. Éd. J.-L. Fournet, « Disposition et réalisation graphique des lettres et des pétitions protobyzantines : pour une paléographie "signifiante" des papyrus documentaires ", Proceedings of the

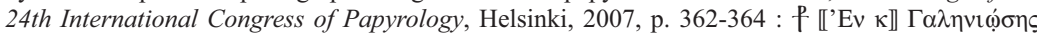

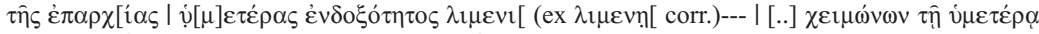

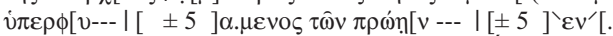

51. Voir J. Gascou, Fiscalité et société en Égypte byzantine, Paris, 2008, p. 431-439. 


\section{Les pièces fiscales}

Cet ensemble compte une vingtaine de documents, dont un conservé à la Bibliothèque nationale de France. Il s'agit surtout de reçus d'impôts pour des fournitures aux cavaliers « Maures » de la garnison locale et autres corps de troupes. Parfois émis par le

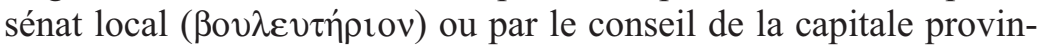
ciale d'alors, Antinoopolis, ils témoignent ainsi, contre les vues de certains historiens, d'une vie municipale encore forte.

\section{LES DOCUMENTS ÉCONOMIQUES}

Il s'agit surtout d'ordres de paiement, de reçus de loyers, de baux de cheptel, de terres agricoles et d'immeubles d'habitation ou d'ateliers, comme des huileries ou des ateliers de potier. Ces types documentaires sont déjà attestés, mais nous saisissons un style notarial et des formules propres à notre cité, des usages monétaires singuliers, des particularités économiques : ainsi les baux d'ovins étaient jusqu'à présent assez rares et il est bien probable que leur présence dans le lot de l'Académie soit à mettre en rapport avec l'artisanat textile local (P.Acad. inv. 24 et 39/1).

Voyons à présent les données sociales en cause. Ces pièces proviennent surtout d'un milieu caractéristique du Bas-Empire, la bourgeoisie de la fonction publique militaire et civile. Nous avons ainsi deux familles de l'aile de cavalerie des Mauri Scutarii stationnée à Lycopolis ${ }^{52}$, en particulier celle d'un certain Némésiôn fils de Maximos, dont on suit la lente et presque machinale carrière, selon tous les grades de la cavalerie, dans la deuxième moitié du $\mathrm{V}^{\mathrm{e}}$ siècle, depuis celui de simple recrue vers 454 (P.Acad. inv. 66/2), puis draconarius, puis biarchus, puis centenier. Il devint ducenier en 471/472 (ou 485/486) selon P.Acad. inv. 76/1. Il fut enfin détaché dans les bureaux militaires provinciaux (P.Acad. inv. 37/2). Sa fortune consistait en maisons de rapport, terres agricoles, vignobles et palmeraies. Une bonne part de ces biens était concentrée dans le village lycopolitain d'Imouthès (peu attesté jusqu'à présent), mais s'étendait aussi à la cité voisine d'Apollonopolis Parva (P.Acad. inv.

52. Une unité sœur de cette aile se trouvait à la même époque dans la ville voisine d'Hermopolis. Pour une actualisation de son dossier, B. Palme, « Spätrömische Militärgerichtsbarkeit in den Papyri ", Symposion 2003, Vienne, 2006, p. 401, environ 40 pièces ; une délimitation exacte est en fait difficile. Pour les quelques attestations de la branche lycopolitaine n'appartenant pas à nos archives, voir P.Oxy. LXXII 4893, 4. 
65/1). Le père de Némésiôn était déjà militaire (P.Acad. inv. 49/2) et a dû lui faciliter la carrière, à moins que cette hérédité n'ait même rendu ce genre de carrière inévitable. Nous avons aussi un texte relatif aux affaires de sa femme, Bictorinè, qui mourut bien avant lui (P.Acad. inv. 66/2). De bonne famille, cette dame possédait au moins une maison et un bateau (P.Acad. inv. 59/2).

Le milieu des fonctionnaires civils est illustré par les papiers d'un greffier ou sténographe (exceptor) de l'administration provinciale nommé Polycratès, fils d'un comptable de la même instance (numerarius) appelé Thômas (encore une sorte de « pistonné» ou d'héritier forcé des fonctions de son père). Polycratès et sa femme apparaissent surtout comme contribuables pour les annones militaires, mais, d'après un reçu de loyer (P.Acad. inv. 47), Polycratès était aussi un possédant et, comme dans le cas de Némésiôn, ses propriétés s'étendaient à la cité voisine d'Antaiopolis. Il y exploitait aussi des terres pour le compte d'autres propriétaires, comme un certain Panolbios, conseiller municipal ou curiale d'Antaiopolis (P.Acad. inv. 55) connu par d'autres archives de la région, qui situent son floruit au début du $\mathrm{VI}^{\mathrm{e}}$ siècle.

D'autres pièces nous reportent dans une ambiance ecclésiastique avec une série de reçus et ordres de paiement au nom des évêques Sarapiôn (P.Acad. inv. 45/1 ; 46/1-3) et Phoibammôn (P.Acad. inv. 77/1-6), qui complètent ainsi des fastes peu remplis. Certaines même émanent de milieux monastiques.

Ces documents, comme le prévoyait au reste Seymour de Ricci, apportent maintes données historiques nouvelles sur les institutions militaires, l'onomastique lycopolite, la toponomastique, en particulier les institutions religieuses chrétiennes (sanctuaires de l'archange Michel $^{53}$, de saint Sarapiôn ${ }^{54}$, de saint Cyriaque ${ }^{55}$, diverses églises de village). Certains de ces renseignements sortent parfois de l'ambiance locale et touchent à l'histoire et aux institutions de l'Empire.

Voyons en particulier quelques apports de ce lot à l'histoire religieuse : dans une location de maison (P.Acad. inv. 69), datée de 420 contractée auprès d'un de nos militaires par un certain Agésilaos, le bornage de l'immeuble loué fait état d'un ci-devant temple (l'expression grecque pote hieron) dont le nom ou surnom pourrait faire allusion au phénix. Presque trente ans après l'interdiction des 
rites païens, les temples survivent donc dans la toponymie et, selon toute probabilité, encore qu'abandonnés, sont toujours debout.

Quant à l'histoire des institutions centrales, signalons l'apport P.Acad. inv. 62, un contrat de boulangerie. Ce document, daté de 534 , sous le règne de l'empereur Justinien, est d'un type assez peu commun. Un boulanger d'un hameau de la cité d'Antaiopolis (désigné du nom très local d'ỏ $\rho \tau о \kappa о \lambda \lambda \eta \tau \eta \varsigma^{56}$ ) s'engage à préparer le pain des employés locaux des domaines impériaux, techniquement la domus divina. L'organigramme administratif des domaines de Justinien y est décrit avec des détails que nous n'avions pas jusqu'à présent, car nos sources étaient surtout constantinopolitaines.

Son édition est donnée ci-dessous en Appendice 3.

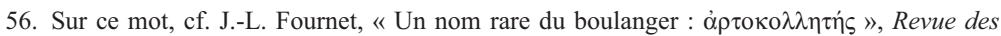
études grecques 113, 2000, p. 392-412. 

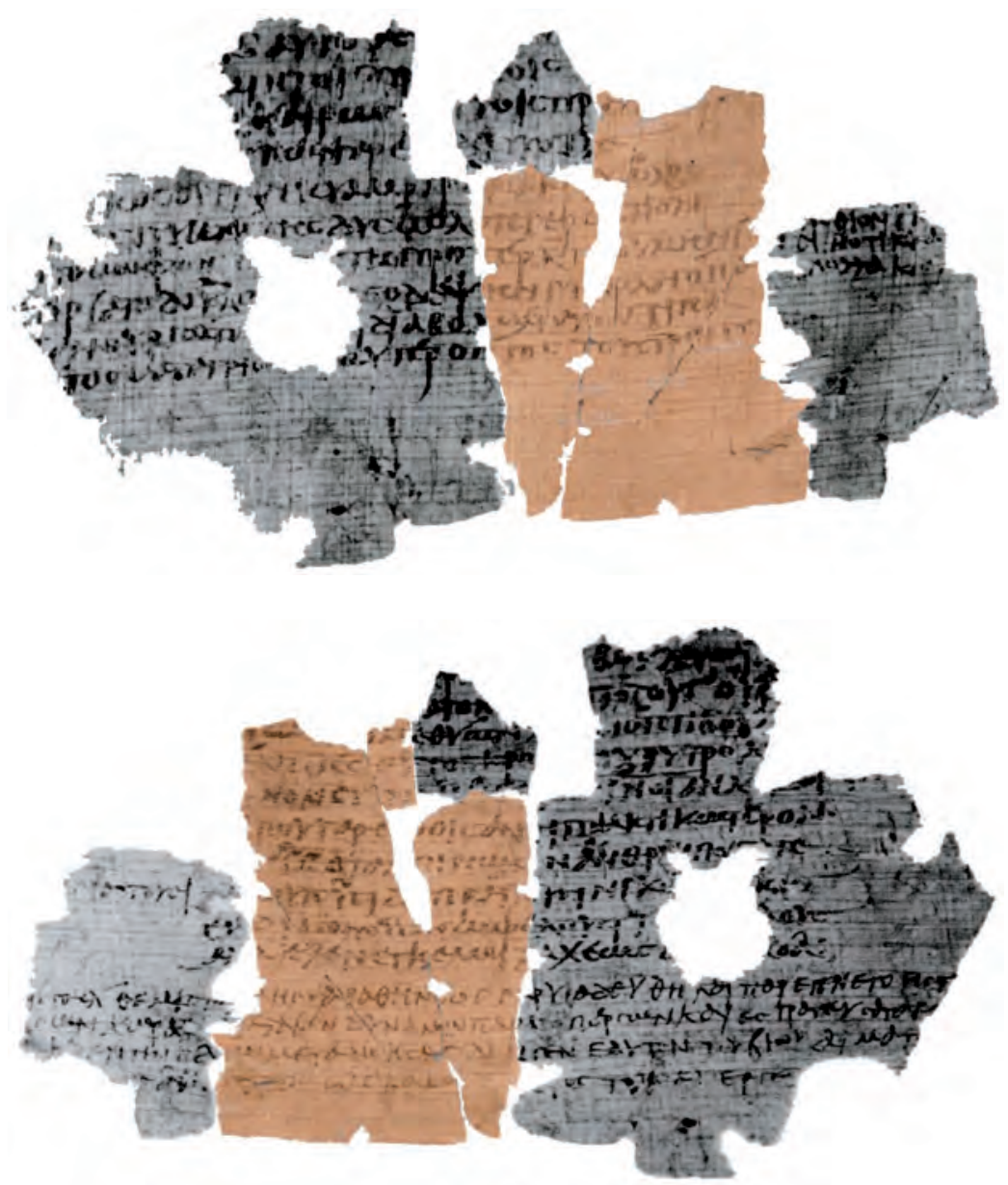

Figs. 7-8. - Le feuillet d'Aristophane, Cavaliers, constitué de P.Acad. 3/4 (en couleurs) et de Bodl. Ms. Gr. class. f. 72 (P) (en noir et blanc) [images tirées de CLGP Pars I, vol. 1, fasc. 4, tab. I-II]. 


\section{APPENDICES}

\section{Aristophane, Cavaliers, 37-46, 86-95, avec scolies}
A : $\mathrm{H} \mathrm{7,7} \mathrm{x} \mathrm{L} \mathrm{6,6} \mathrm{cm}$
B : H 2,1 x L $1,9 \mathrm{~cm}$
C : H 4 x L $3 \mathrm{~cm}$
D : H $6 \times$ L $4,4 \mathrm{~cm}$

P. Acad. 3/4 + Bodl. Ms. Gr. class. f. 72 (P)

Lycopolis

fin $I V^{\mathrm{e}} /$ déb. $V^{\mathrm{e}} \mathrm{s}$.

Fig. 7-8

Bibl. : B.P. Grenfell et A.S. Hunt, "Some Classical Fragments from Hermopolis », Mélanges Nicole, I, Genève, 1905, p. 212-217 (éd. de Bodl. gr. class. f. $72(\mathrm{P}))$.

J.-L. Fournet et F. Montana, « [Aristophanes], P.Acad. inv. 3 d + P. Bodl. Ms. Gr. class. f. 72 (P), Note marginali a Eq. 37-46, 86-95» dans G. Bastianini, M. Haslam, H. Maehler, F. Montanari et C. Römer (éd.), Commentaria et Lexica Graeca in Papyris Reperta, Pars I, vol. 1, fasc. 4, Munich, 2006, p. 48-56 (édition des scolies ; on y trouvera une bibliographie complète sur les scolies).

Descr. : ce papyrus est actuellement en quatre morceaux. P.Acad. 3/4 (= fr. D, résultant lui-même du raccord de quatre fragments) se situe à droite de Bodl. Ms. Gr. class. f. 72 (P), fr. A et B, auxquels il est jointif. Bord inférieur d'origine.

Main : informelle (cf. Cavallo et Maehler, Greek Bookhands, p. 28), datée entre 350 et 450 par les premiers éditeurs, de la seconde moitié du IV siècle par Cavallo et Maehler, l. c. La main des scolies (m2) serait, d'après E. Lobel (ap. G. Zuntz, Die Aristophanes-Scholien der Papyri, $2^{\mathrm{e}}$ éd., Berlin 1975, p. 29), sensiblement plus récente $\left(V^{\mathrm{e}} \mathrm{s}\right.$.) ; mais, en l'absence d'informations sur l'âge du scripteur, on ne peut être aussi affirmatif et il est possible que ces scolies, écrites dans un «style » plus récent, soient à peu près contemporaines du texte principal.

Le raccord entre le fragment P.Acad. 3/4 et Bodl. gr. class. f. 72 (P), publié il y a un siècle par B.P. Grenfell et A.S. Hunt ${ }^{57}$, soulève un problème de provenance qui a été évoqué plus haut ${ }^{58}$.

Les quatre fragments conservent la partie inférieure d'un folio de codex. Chaque page contenait 49 lignes de texte ${ }^{59}$, auxquelles venaient s'ajouter dans les marges latérales, inférieures (et peutêtre supérieures) des scolies écrites postérieurement par une autre

57. Il est enregistré dans le Mertens-Pack ${ }^{3}$ sous le 141. L'ensemble des fragments gardera le même numéro.

58. Cf. supra, p. 1045-1046.

59. Et non 40 d'après E. Turner, Typology of the Early Codex, Philadelphia, 1977, p. 102, n. 12 . 
main. La surface du texte de première main devait être d'une hauteur d'environ 22,5 et d'une largeur variable (entre 9 et 10,8 cm, d'après les mesures extrêmes relevées). Ce qui reste des marges permet de reconstituer un feuillet d'environ $\mathrm{H} 28 / 29$ x L $18 \mathrm{~cm}$, qui correspond au groupe 5 de Turner, caractéristique de manuscrits des $I V^{e}-V^{e}$ s. ${ }^{60}$

L'écriture est très proche de la cursive contemporaine (par exemple, P.A thm II 145, lettre privée), ce qui est l'indice que nous avons affaire avec ce codex à une copie privée.

Les signes diacritiques employés dans le texte proprement dit sont l'apostrophe (qui semble systématiquement marquée), le point en haut ${ }^{61}$ (qui est mis assez systématiquement pour marquer les fins de phrase ${ }^{62}$ ) et le double point (signalant un changement de personnage $)^{63}$, les signes de la longue $(-)^{64}$ et de la brève $(-)^{65}$ - marqués sans qu'ils soient vraiment utiles ${ }^{66}$-, les esprits doux $\left({ }^{-1}\right)^{67}$ et rude $\left({ }^{-}\right)^{68}$ et les accents aigu ${ }^{69}$ et circonflexe (de deux formes : majoritairement ${ }^{-}$; une fois ${ }^{\sim 70}$ ). À l'exception des apostrophes, de première main, ces signes diacritiques sont pour la plupart d'une encre plus foncée, indice qu'ils ont été ajoutés par une autre main ou en tout cas ultérieurement, comme c'est habituel dans les papyrus littéraires.

Le texte ne donne qu'une seule variante sans intérêt (v. 87) ${ }^{71}$.

L'intérêt de ces fragments tient à la présence de trois scolies marginales, copiées dans une écriture plus menue et plus fine mais assez similaire à celle du texte. Ce papyrus fait partie d'un ensemble très significatif, d'époque surtout tardive, de papyrus annotés d'Aristophane dans lequel les Cavaliers sont très bien représentés ${ }^{72}$. La scolie au v. 41 ne correspond à aucune de celles transmises par

60. E. Turner, op. cit., p. 16-17. On trouvera le détail de la reconstitution des folios dans J.-L. Fournet et F. Montana dans Commentaria et Lexica Graeca in Papyris Reperta, Pars I, vol. 1, fasc. 4 , p. 49.

61. Ou à mi-hauteur au v. 91 .

62. Sauf à la fin des v. 43, 90 et 94.

63. V. 95. Cf. note ad loc.

64. V. $43,88,93$.

65. V. 45 .

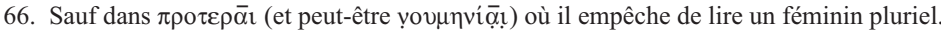

67. V. 87.

68. V. 89,92 .

69. Deux fois fautifs : v. 40 et 46.

70. V. 94 (cf. note ad loc.).

71. La variante du v. 92 est une coquille de l'édition de Grenfell et Hunt (cf. note ad loc.).

72. Cf. supra, p. 1052. On a deux autres éditions pourvues de scolies marginales des Cavaliers: P.Bingen 18 (= Mertens-Pack ${ }^{3}$ 142.1), IV ${ }^{\mathrm{e}}$ siècle ; Berliner Klassikertexte IX 5 (= Mertens-Pack ${ }^{3}$ $142), V^{\mathrm{e}}$ siècle. À titre indicatif, les autres comédies du même auteur les mieux attestée en édition pourvues de scolies sont Le Ploutos (3 ou 4) et La Paix (3). 
la tradition ${ }^{73}$. Celle au v. 93 en est en revanche très proche ${ }^{74}$. La troisième (ad $\Theta \varepsilon \mu 1 \sigma \tau о \kappa \lambda \varepsilon ́ o v \varsigma$, v. 84), plus substantielle, a fait l'objet d'une étude approfondie par G. Zuntz, Die Aristophanes-Scholien

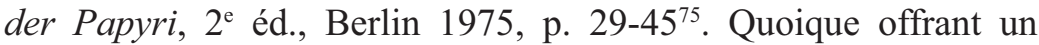
certain nombre de divergences, elle s'apparente à un résumé très condensé de la première version de la scolie ancienne (I dans l'éd. de Jones, p. 30) et, d'après Zuntz, serait une rédaction parallèle dérivant, à travers deux intermédiaires, de l'historien Aristodème (dont le floruit n'est pas postérieur au II ${ }^{\mathrm{e}} \mathrm{s}$. apr. J.-C.) ${ }^{76}$. Pour une étude de ces scolies, le lecteur se reportera à l'édition que J.-L. Fournet et F. Montana en ont donnée dans les Commentaria et Lexica Graeca in Papyris Reperta, Pars I, vol. 1, fasc. 4, Munich, 2006, p. 48-56 (abrégé $C L G P$ dans les notes de commentaire ci-dessous).

Le texte est édité en transcription semi-diplomatique; le texte des scolies est données dans les notes en transcription normalisée (avec esprits et accents). Les remarques paléographiques sur les fragments de la Bodléienne que l'on trouvera dans les notes de commentaire sont empruntées directement à l'édition de Grenfell et Hunt (abrégés G.-H.).

Le texte a été collationné sur l'édition de V. Coulon et H. Van Daele (C.U.F.), utilisée pour les variantes, et celle de A.H. Sommerstein.

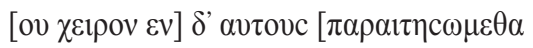

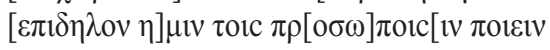

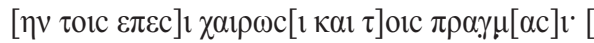

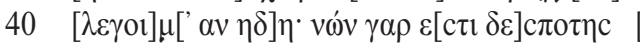

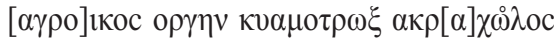

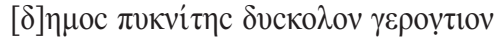

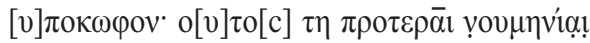
(m2) $\alpha[$ (m2) [ кианоис]

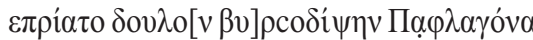

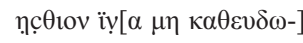

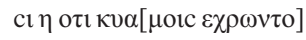

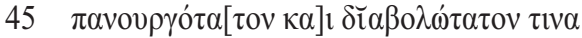

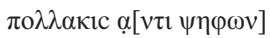

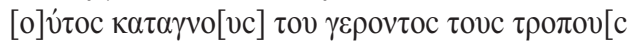

73. Prolegomena de comoedia. Scholia in Acharnenses, Equites, Nubes : Scholia vetera in Aristophanis Equites, éd. D.M. Jones et Scholia Tricliana in Aristophanis Equites, éd. N.G. Wilson, (Scholia in Aristophanem, I, 2, Groningen-Amsterdam, 1969).

74. Cf. note ad loc.

75. Rééd. de G. Zuntz, " Die Aristophanes-Scolien der Papyri », Byzantion 13, 1938, p. 659-677.

76. Cf. note ad loc. 


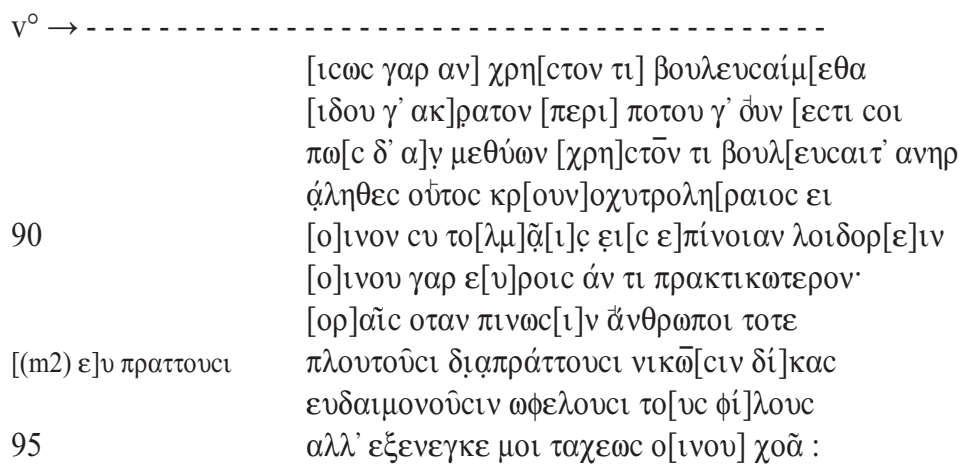

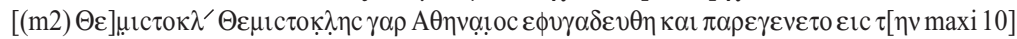

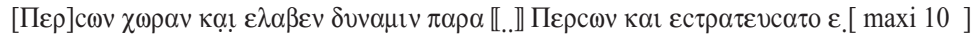

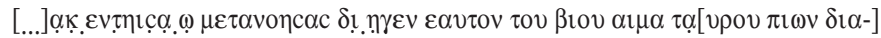

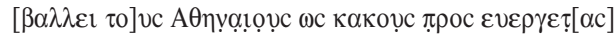

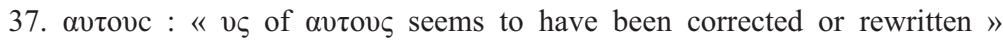
(G.-H.).

40. vต́v : pour vต̂tv. «The final $v$ of $v \omega v$ has been corrected from $\mathrm{t}$ by the second hand, which has added an accute accent (apparently) over the $\omega$; cf. 1. 46, note » (G.-H.).

scol. 41. $\alpha$ [ : cf. CLGP, p. 50. F. Montana propose, sans certitude, d'y voir le

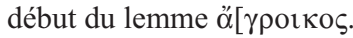

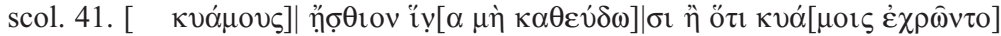

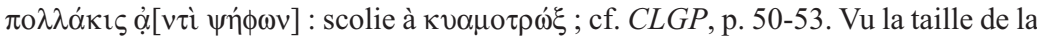

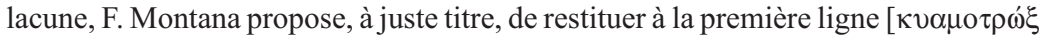

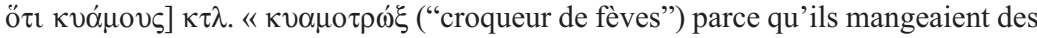
fèves afin de ne pas s'endormir ou parce qu'ils usaient souvent de fèves comme de bulletins de vote $»$.

41. $\alpha \kappa \rho[\alpha] \chi \omega \omega_{0} \lambda \mathrm{oc}: \alpha \kappa \rho[\alpha] \chi \omega \lambda \mathrm{oc}$ corrigé à juste titre en $\alpha \kappa \rho[\alpha] \chi 0 \lambda \mathrm{oc}$.

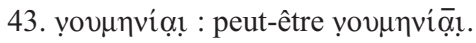

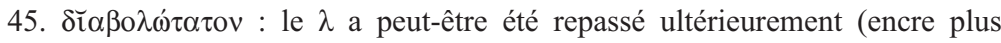
noire).

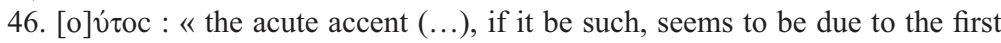
hand. For an other example of an acute instead of a circumflex accent, cf. 1.40 » (G.-H.).

scol. 84 cf. post 95 .

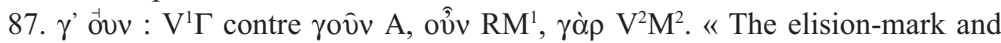
smooth breathing above ov seem to be due to the first hand; but the $v$ has certainly been corrected by the second hand, apparently from $\varepsilon$, so that it is probable that the first hand wrote $\gamma^{\prime} \varepsilon c \tau \iota$ » (G.-H.).

88. $\alpha]$ : : les traces correspondraient plus à un $\rho$ ou o qu'à un $v$.

$[\chi \rho \eta] c \tau \bar{o} v:$ : there is a short horizontal stroke above the o, similar to that found above the $\omega$ of vik $\omega \sigma v v$ in 1. 93. This is more like the sign indicating a long vowel than anything else, but, though the sign for a short vowel occurs in 1. 45, the insertion of a mark of quantity over ov of $\chi \rho \eta \sigma \tau o v$ and $\omega$ of $v \iota \kappa \omega \sigma \iota$ is out of place " (G.-H.). 


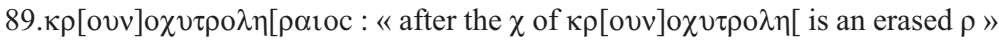
(G.-H.).

90. $\varepsilon] \pi i v o r \alpha v$ : « above ] $\pi$ lvor is a long horizontal stroke, which seems to be accidental » (G.-H.).

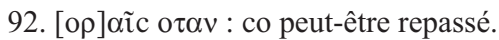

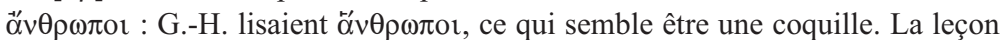
des mss est d'ailleurs unanimement $\alpha$ $v \theta \rho \omega \pi$ or.

93. vıк̄̄ $[\mathrm{c} \iota v$ : pour le trait, cf. v. 88 n. à $[\chi \rho \eta] c \tau \bar{v}$.

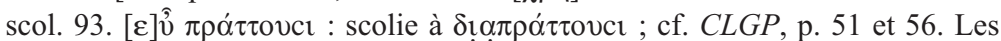

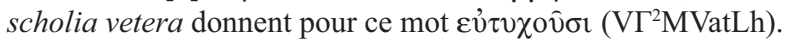

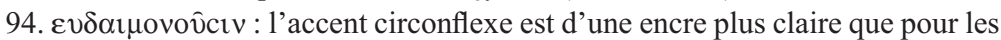
autres accents; il a en outre une forme différente des autres circonflexes.

95. $\alpha \lambda \lambda$ ': un point d'encre semble précéder l'apostrophe.

$\chi 0 \tilde{\alpha}:$ : the circumflex above the $\alpha$ of $\chi 0 \alpha$ and the double point after it were probably inserted by the writer of the scholia. The double point ought to indicate a change of speaker, as e. g. in the Lysistrata fragment. Perhaps line 96 tòv vov̂v

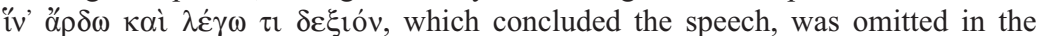
papyrus » (G.-H.). Cf. J.C.B. Lowe, « The Manuscript Evidence for Changes of Speaker in Aristophanes ", Bulletin of the Institute of Classical Studies 9, 1962, p. 27-42 (p. 31-35 pour les papyrus).

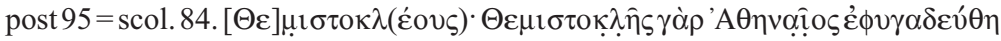

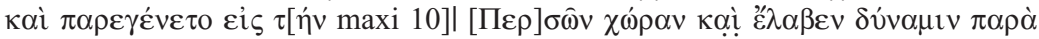

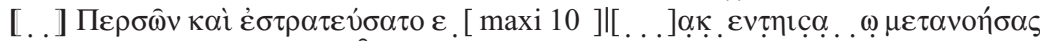

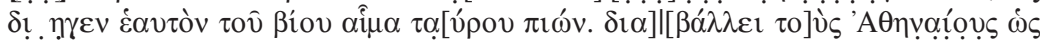

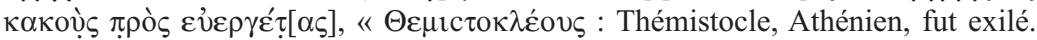
Parvenu à $[\ldots]$ territoire des Perses, il reçut des Perses une armée et mena une expédition militaire contre [...]. S'étant ravisé, il s'ôta la vie en buvant du sang de taureau. Il accusait les Athéniens de mal se comporter envers leurs bienfaiteurs ». Scolie à $\Theta \varepsilon \mu 1 \sigma \tau о \kappa \lambda \varepsilon ́ o v \varsigma$ (v. 84), écrite dans la marge inférieure car trop longue pour tenir dans la marge latérale. Sur cette importante scolie que le fragment de l'Académie permet de mieux lire, en corrigeant les restitutions de G.-H., reprises par Zuntz, cf. CLGP, p. 51 et 53-56. Le texte comporte encore quelques incertitudes :

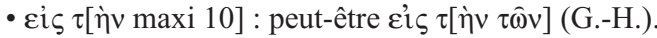

- $\dot{\sigma} \sigma \rho \alpha \tau \varepsilon v ́ \sigma \alpha \tau o \varepsilon$. [ maxi 10 ] : devant la lacune peut se lire indifféremment $\varepsilon i ̣[\varsigma$

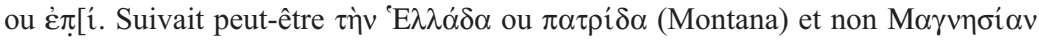
(G.-H., Zuntz) qui impliquerait qu'il s'agisse de l'objectif de l'expédition.

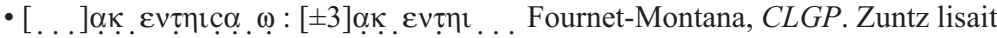

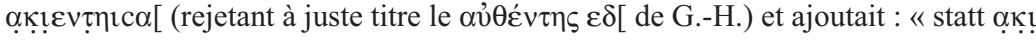

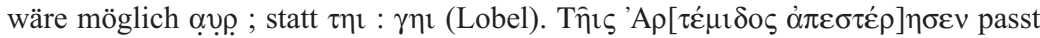

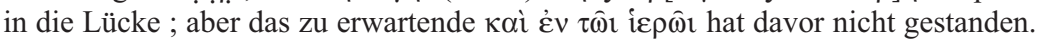
Auch mit $\dot{\varepsilon} v \gamma \hat{\eta} \imath \sigma \alpha$ [ wüsste ich nichts anzufangen; denn was sollte man etwa mit

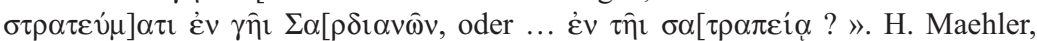
qui a bien voulu contrôler l'original de la Bodléienne à la demande de Fournet,

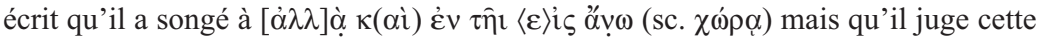
solution douteuse dans la mesure où les sources ne disent pas que Thémistocle se suicida « dans le territoire au-dessus (de Magnésie) ». La lecture $[\dot{\alpha} \lambda \lambda] \grave{\alpha} \kappa(\alpha i ́)$ au début de la ligne reste néanmoins une solution très séduisante. G. Bastianini, de

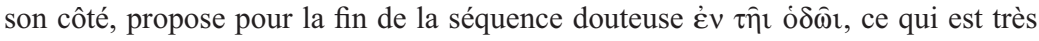


séduisant pour le sens (« en chemin, changeant d'avis, etc. »). Mais il est difficile de lire o. Le ductus de la lettre semble incompatible avec un o mais correspond parfaitement à un c (ce qui est confirmé par H. Maehler). Quant au $\delta$, il laisse dubitatif : il ne ressemble pas aux autres $\delta$ de la seconde main dans lesquels la barre oblique est à peine incurvée à son extrémité gauche alors qu'ici elle l'est très nettement. En revanche, cette lettre est très similaire au premier alpha de $\alpha \theta \eta v ̣$ țọ̣c de la 1. 4. On serait donc tenté de lire ça. La fin du mot sur le fragment de Paris est très effacée,

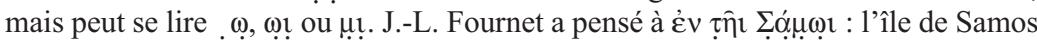
est tout près de Magnésie, mais, malheureusement, aucune source, à notre connaissance, ne fait mourir Thémistocle à Samos. Notons par ailleurs, aussi bien au sujet

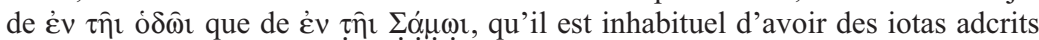
dans des scholies copiées au IV/V $\mathrm{V}^{\mathrm{e}}$ siècles ; mais il est vrai que l'on peut objecter quelques contre-exemples (Berliner Klassikertexte IX 15, note au v. 574 et MPER III 20, $\mathrm{r}^{\circ} 1$, tous deux du $\mathrm{V}^{\mathrm{e}} \mathrm{s}$., cités dans $C L G P$, p. 55).

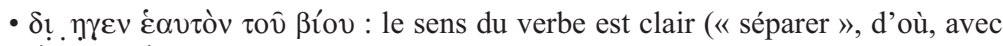

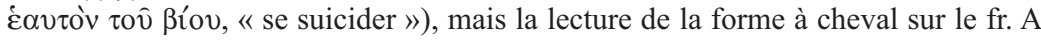
et $\mathrm{D}$ est difficile. Deux solutions viennent à l'esprit : (1) $\delta$ ' $\dot{\xi} \xi \hat{\eta} \gamma \varepsilon v$ pour $\dot{\varepsilon} \xi \dot{\eta} \gamma \alpha \gamma \varepsilon v$, proposé par F. Montana qui l'étaye par d'excellents parallèles (cf. CLGP, p. 55-56). Mais, après le delta, il y a un trait vertical qui, par sa verticalité, me semble assez difficilement compatible avec un epsilon, ailleurs toujours penché ou arrondi. De plus, on devrait voir l'amorce des hastes horizontales ou obliques, ce qui n'est absolument pas le cas. Entre cette lettre, qui ressemble plutôt à un iota, et le $\eta$, il y a un point d'encre infime très légèrement sous la ligne, mais pas assez de place pour un

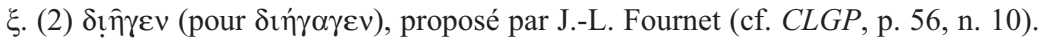
Mais, si elle est défendable sémantiquement, la lecture fait difficulté dans la mesure où il y aurait un espace anormalement large entre le 1 et le $\eta$ et que le point d'encre entre ces deux lettres resterait inexpliqué.

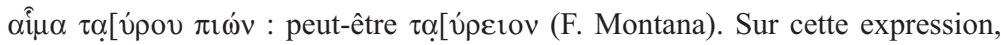
cf. D. Arnould, " "Boire le sang de taureau" : la mort de Thémistocle », Revue de Philologie 67, 1993, p. 229-235.

\section{Concordance entre calendriers égyptien, julien et macédonien}
P.Acad. inv. $1 \mathrm{r}^{\circ}$
H $10,8 \times$ L $17,7 \mathrm{~cm}$
$\mathrm{V}^{\mathrm{e}} \mathrm{s}$.
Lycopolis
Fig. 9

Descr. : au dos du feuillet est écrite, transversa charta, une pétition, partiellement perdue lorsqu'on a rogné le feuillet en cadrant sur le texte sur le recto. (fig. 10)

Main : même main que la pétition écrite sur l'autre face ainsi que la recette médicale P.Acad. inv. 6/1 et P.Acad. inv. 49.

Ce texte est une concordance entre trois calendriers : égyptien (col. I), julien (col. II) et macédonien (col. III). Quoique les noms de mois égyptiens occupent la première colonne, cette concordance prend comme référence le calendrier julien : elle commence par le 


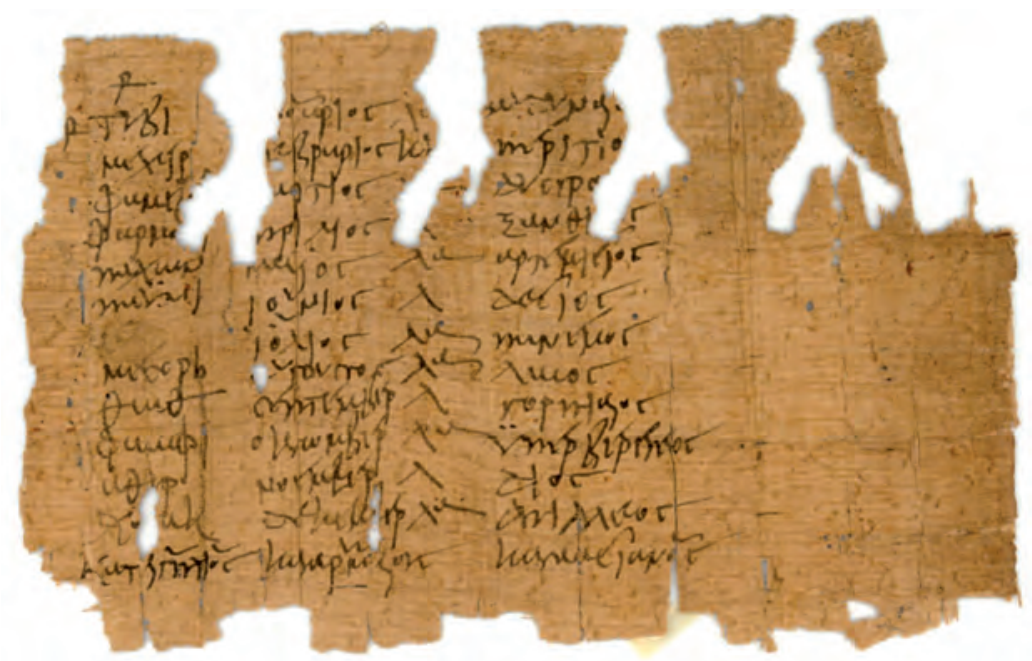

FIG. 9. - Concordance calendaire (P.Acad. inv. $1 \mathrm{r}^{\circ}$ ).

mois de janvier et se termine par décembre, alors que l'année égyptienne débute avec Thôth pour finir avec Mesorên ${ }^{77}$. C'est la première concordance sur papyrus donnant ces trois calendriers ${ }^{78}$.

Les noms de mois latins (qui sont simplement translittérés ${ }^{79}$ ) sont accompagnés de chiffres donnant le nombre de jours que contient

77. Sur cet ordre, cf. J. Rea, «A New Bithynian Month List », Zeitschrift für Papyrologie und Epigraphik 90, 1992, p. 289.

78. Les textes les plus proches sont :

- P. Iand. inv. 654 (éd. H.G. Gundel, Archiv für Papyrusforschung 16, 1958, p. 13-19), $\mathrm{VI}^{\mathrm{e}} / \mathrm{VII}^{\mathrm{e}} \mathrm{s}$. : calendriers julien et égyptien.

- P. Berol. 1080 (éd. G. Poethke, Archiv für Papyrusforschung 46, 2000, p. 160), IVe/V s. : calendriers julien et égyptien.

- P. Fay. $135 \mathrm{v}^{\circ}$ descr. (éd. J. Kramer, Glossaria bilinguia in papyris et membranis reperta, Bonn, 1983, n ${ }^{\circ} 11$, p. 77-81), IV $\mathrm{s}$. : calendriers julien et égyptien.

- P. Paris 4 (= Mertens-Pack ${ }^{3}$ 2332, Cribiore 98$)$, II $^{\mathrm{e}}$ S. av. : calendriers attique et macédonien.

- P. Rain. Cent. 31 (éd. C. Grassien, Tyche 12, 1997, p. 69-70), $\mathrm{VII}^{\mathrm{e}} / \mathrm{VIII}^{\mathrm{e}} \mathrm{s}$. : calendrier égyptien, calendriers julien-égyptien et cappadocien-égyptien.

- Eine alexandrinische Weltchronik, éd. A. Bauer et J. Strzygowski, Vienne, 1905, p. 18, ve s. : calendriers hébreu, égyptien et athénien.

- D. Montserrat, "A Fragment of a Monastic Duty Roster? ", Bulletin of the American Society of Papyrologists 27, 1990, p. 163-168 (corrigé par J. Rea, Zeitschrift für Papyrologie und Epigraphik 90, 1992, p. 289-291), $\mathrm{VII}^{\mathrm{e}} \mathrm{s}$. : calendriers égyptien et bithynien.

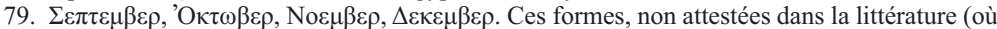

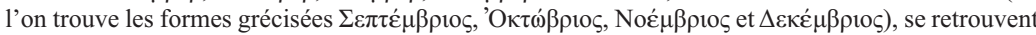
dans une concordance de même genre conservée par le P.Fay. $135 \mathrm{v}^{\circ}$ (= J. Kramer, Glossaria bilin-

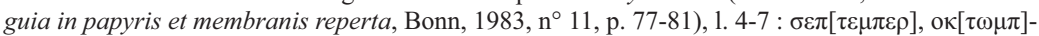

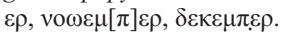




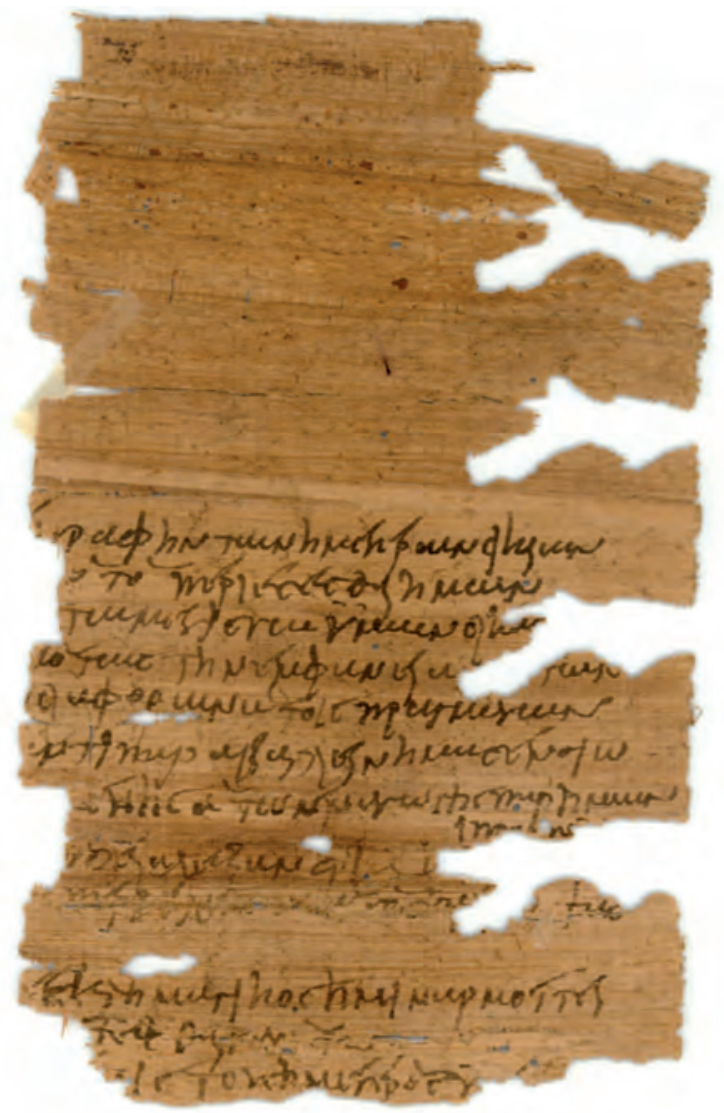

FIG. 10. - Pétition au verso de P.Acad. inv. 1.

chacun, puisque celui-ci présente une alternance de mois de 30 et 31 jours - sans compter le mois de février de 28 jours - étrangère aux deux autres systèmes ${ }^{80}$. On remarquera que les mois de septembre, octobre, novembre et décembre ont été purement et simplement translittérés, sans désinences grecques en -los comme c'est l'usage.

Sur la fonction de ce texte, voir supra p. 1053-1054.

80. Une autre liste de mois précise pareillement le nombre de jours : il s'agit d'un papyrus de Strasbourg édité par J.-L. Fournet, " Nouveaux textes scolaires », Bulletin de l'Institut français d'archéologie orientale 101, 2001, p. 167 (= Mertens-Pack ${ }^{3}$ 2749.02). Dans ce cas, toutefois, il n'est question que des mois égyptiens mais la partie droite du papyrus, en lacune, donnait peut-être la correspondance avec un autre calendrier. 


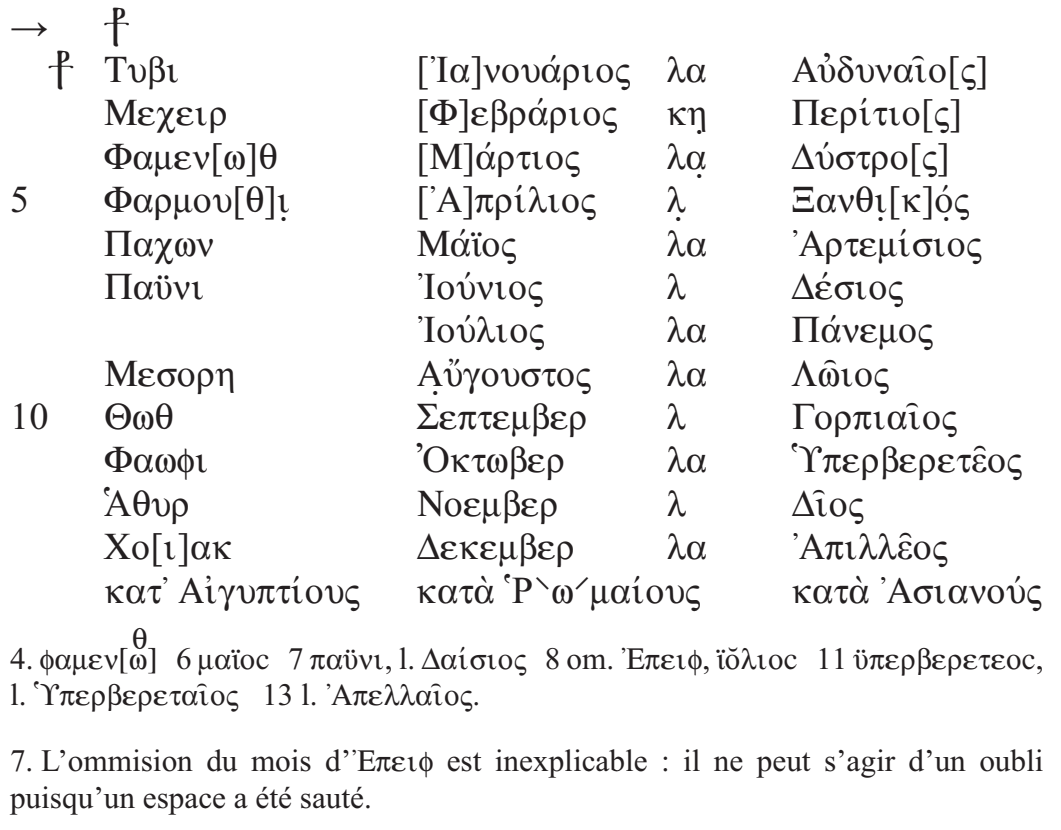

\section{Contrat de boulangerie}

P.Acad. inv. 62

H 30,4 x L17,3 cm

11 avril 534

Terythis (Antaiopolite)

Fig. 11-12

Comme d'autres pièces du dossier, ce document provient de l'Antaiopolite. Dans ce contrat écrit dans une cursive fine et légèrement penchée, amputé de plus des deux tiers de sa partie inférieure à gauche et de deux lignes en bas, le boulanger Aurelius Phèus, du hameau de Pelaiouae ${ }^{81}$ du village antaiopolite de Terythis, s'engage auprès de la domus divina de Justinien à boulanger $(\pi \varepsilon ́ \psi \alpha \imath)$ du pain pour des « dépendants » de cette instance ${ }^{82}$. Nous ne connaissions pas de convention analogue ${ }^{83}$, ce qui rend d'autant plus regrettables les pertes textuelles, du point de vue du contenu et des formes juridiques. En l'état, cette pièce intéressera surtout les historiens

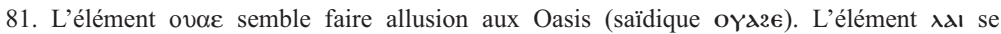
rencontre comme formant de toponymes locaux.

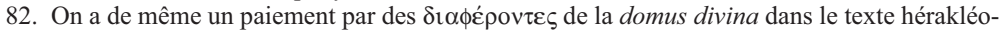
polite P.Vindob.Tand. 18,30 (interprété « von Beamten, die den kaiserlichen Domänen verbunden sind $\gg)$.

83. A. Jördens, dans son P.Heid. V, consacré au régime contractuel des relations de travail, n'en signale pas, du moins pour l'époque byzantine. 
des institutions de la domus divina. On notera, 1. 7, à la tête des domaines impériaux, un collège de deux arabarques. Sans parler de l'étymologie du $\operatorname{mot}^{84}$ ni de la forme alternante $\dot{\alpha} \lambda \alpha \beta \alpha \dot{\alpha} \rho \chi \eta \varsigma^{85}$, ni de l'utilisation occasionnelle de ce mot comme nom propre $^{86}$, le statut et les fonctions de ces agents, qui sont attestés en Égypte depuis l'époque hellénistique et qu'on a aussi dans le reste du monde hellénisé (d'après P.Dura 20,5), ne sont pas encore bien compris encore qu'il soit admis que les compétences de l'arabarque s'étendaient aux portoria du désert oriental douanes ou droits intérieurs ${ }^{87}$. Au Bas Empire, on entrevoit en Égypte un lien entre l'arabarque et le gouverneur militaire provincial, le duc ou parfois le comte du limes. La constitution CTh IV 13, 9 (CJ IV 61,9), adressée en 381 au comte des largesses sacrées indique (sans précisions institutionnelles) que le comte d'Égypte (désignation du gouverneur militaire alternant à l'époque avec celle de $\delta o v ́ \xi)$ était compétent pour un vectigal de l'arabarchie. Comme l'a mis en évidence R. Delmaire, l'arabarque peut lui-même devenir duc : du moins en avons-nous en Thébaïde un cas certain avec Ioannès sous Justin II ${ }^{88}$. C'est pourquoi, et vu la date du texte, nous serions enclins à identifier les présents titulaires de l'arabarchie, Iôannès et Horiôn, aux ducs homonymes qui se sont succédés en Thébaïde quelque temps avant la promulgation de l'Édit XIII par Justinien en $538^{89}$. Comme on le verra encore, la gestion

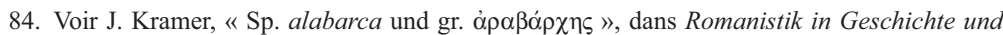
Gegenwart 1, 1995, p. 215-222, après bien d'autres, tient pour une étymologie d'après le nom des Arabes qui, selon lui, étaient souvent chargés de lever les droits de douane et de circulation. Pour une tentative plus récente d'élucidation de l'institution, voir F. Burkhalter-Arce, "Les fermiers de l'arabarchie : notables et hommes d'affaires à Alexandrie », dans Alexandrie : une mégapole cosmopolite, Paris, 1999, p. 41-54.

85. Elle ne se rencontre pas dans les documents égyptiens et n'est sans doute pas authentique. Elle n'est transmise que par des inscriptions non-égyptiennes (où elle peut s'expliquer par des déformations phonétiques) et la littérature où elle peut procéder d'interventions de copistes médiévaux incompréhensifs, par suite d'une fausse étymologie d'après $\alpha ّ \lambda \alpha \beta \alpha$, encre.

86. Cet usage (relevant sans doute d'une onomastique de bon augure) ne complique pas peu l'étude de l'institution (voir O.Eleph.DAIK, $S B$ XXII 15515, 2 (Kom Ombo, patronyme, $B L 11$ : restitution du nom propre 'Ap $\alpha($ ) dans $S B$ XIV 11919, 2 et 4).

87. Pour une mise à jour du dossier, voir F. Morelli, P.Paramone 17, p. 192-193 (tenant compte du présent document). Morelli cite, p. 193, n. 48, une étude d'une impressionnante perspicacité de J. Bingen, « Un nouvel épistratège et arabarque alexandrin », Zeitschrift für Papyrologie und Epigraphik 138, 2002, p. 120, = Pages d'épigraphie grecque II, Bruxelles, 2005, p. 34-36 (ad P. Thomas $10=$ Supplementum Epigraphicum Graecum L 1563). Bingen rappelle le lien fréquent, dans la deuxième moitié du I ${ }^{\mathrm{er}} \mathrm{s}$. entre l'épistratégie et l'arabarchie et voit dans le nouveau titulaire des deux responsabilités qu'il identifie dans l'inscription alexandrine un exemple, entre autres « du rôle du grand capital alexandrin dans la gestion de l'Égypte romaine ».

88. « Le personnel de l'administration financière en Égypte sous le Bas-Empire Romain (IV $-\mathrm{VI}^{\mathrm{e}}$ siècles)», CRIPEL 10, 1988, p. 137 (réf. à P.Cair.Masp. II 67166 et P.Lond. V 1677).

89. Voir Édit XIII § 24. Cette hypothèse a eu l'agrément de R.Delmaire, art. cit., à qui J. Gascou avait communiqué le présent texte. 

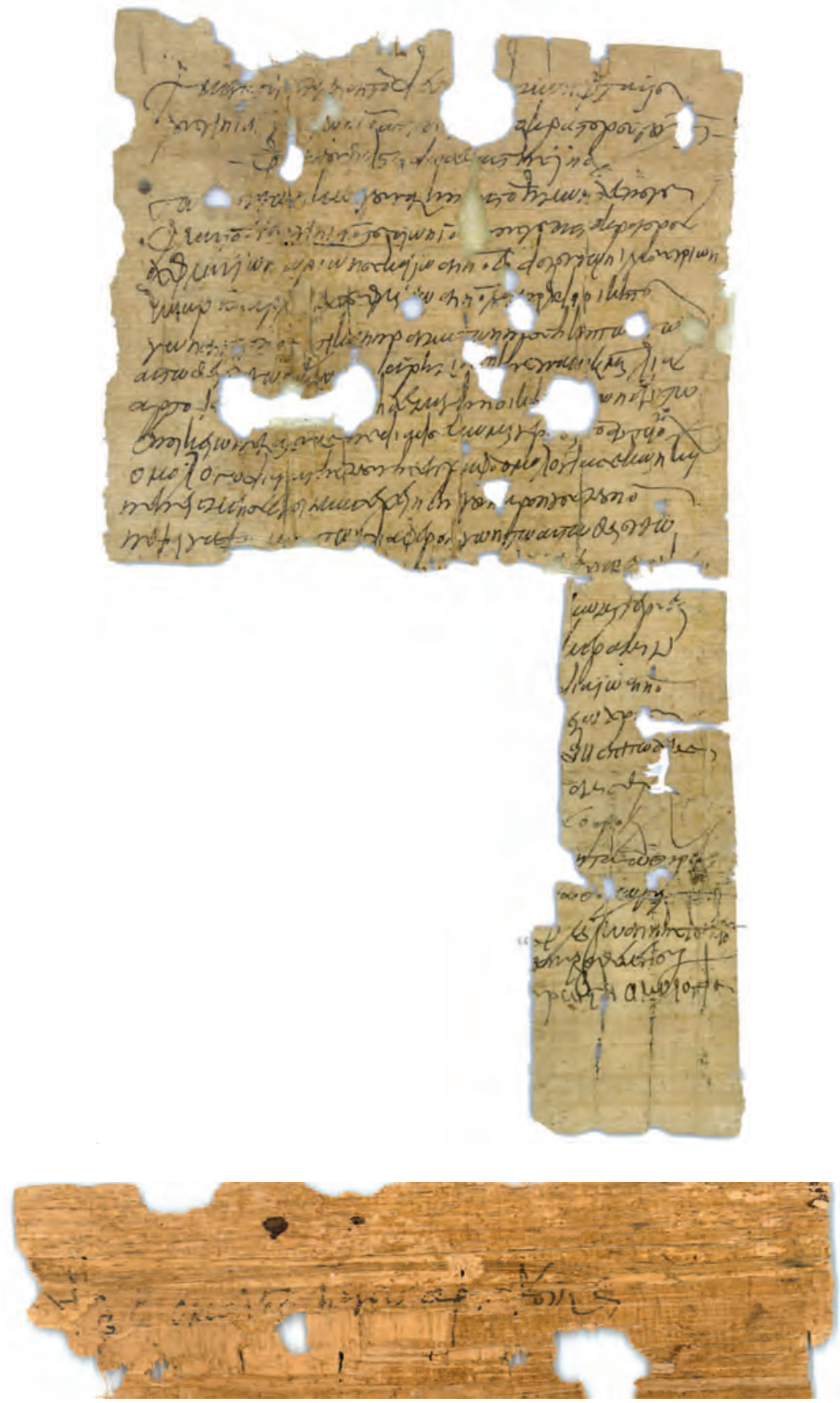

FIGS. 11-12. - Contrat de boulangerie, P.Acad. inv. $62\left(\mathrm{r}^{\circ}\right.$ et $\left.\mathrm{v}^{\circ}\right)$. 
de la domus divina contribue à rapprocher l'arabarque du duc. Nous ignorions jusqu'à ce jour que les arabarques aient, comme ici, assuré leurs fonctions en collège.

On aimerait savoir à quel niveau de l'administration des domaines se placent ces personnages. Le cadre paraît bien égyptien et même thébain. Il semble donc qu'en 534, au dessous de leurs curateurs généraux constantinopolitains, les domus aient disposé, avec les arabarques, d'un relais provincial spécifique. À d'autres époques, le duc était lui-même ce relais ${ }^{90}$. Nous constatons enfin qu'en dernier ressort (mais ce principe administratif était déjà bien enraciné) la domus divina prend en compte la cité, le responsable et subrogé local des arabarques, le clarissime Iôannès de la 1.7 , portant cette fois le nom à fortes suggestions financières de $\delta 101 \kappa \eta \tau \eta \varsigma^{91}$. Le statut de cet agent, plusieurs fois attesté dans notre dossier, n'est pas clair. Fonctionnaire? Notable municipal assumant un munus?

\begin{tabular}{|c|c|}
\hline & f \\
\hline & 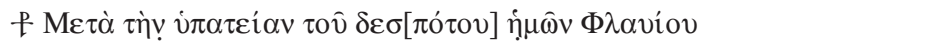 \\
\hline \multirow{6}{*}{5} & 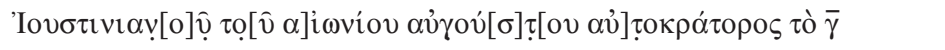 \\
\hline & 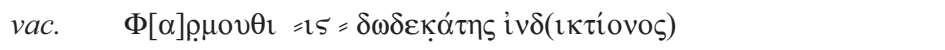 \\
\hline & 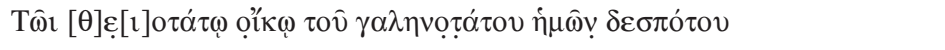 \\
\hline & 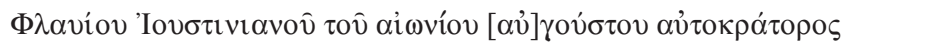 \\
\hline & 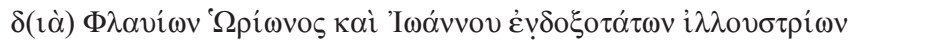 \\
\hline & 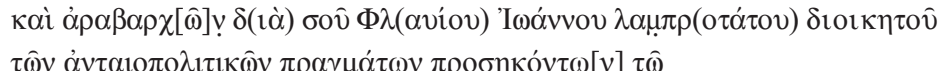 \\
\hline
\end{tabular}

90. J. Gascou, «Les grands domaines, la cité et l’État en Égypte byzantine», Travaux et Mémoires 9, 1985, p. 35, n. 213 (= Fiscalité et société en Égypte byzantine, Paris, 2008, section IX). On trouve aussi à cette place d'anciens ducs (voir L. Tacoma, « Replacement of Parts for an Irrigation Machine of the Divine House at Oxyrhynchus. P. Columbia inv. 83, October 12, AD 549 (?) ». Zeitschrift für Papyrologie und Epigraphik 120, 1998, p. 123-13, à propos d'un certain Théodoros ancien duc et augustal attesté dans ce genre d'attribution sous Justinien par CPR V 18 et P. Col. inv. 83 (= SB XXIV16312; ici collégialement avec son frère l'illustris Théodosios). Tacoma remarque (p. 125, n. 6-7) que Gascou a eu tort de lier statutairement les fonctions de duc et de curateur, cependant la position de Gascou est largement adoptée par F. Morelli, «Zwischen Poesie und Geschichte : Die "Flagornerie" des Dioskoros und der dreifache Dux Athanasios » dans J.-L. Fournet et C. Magdelaine (éd.), Les archives de Dioscore d'Aphrodité cent ans après leur découverte. Histoire et culture dans l'Égypte byzantine, Paris, 2008, p. 237, n. 47 (übliche Verbindung). Pour une attestation littéraire de l'ex-duc Théodoros (indiquant qu'il était d'origine thébaine) remontant aux années 533-536, voir aussi Tyche 9, 1994, p. 21, n. 34 (le texte de Columbia montre qu'il était ancien duc et augustal d'Alexandrie et non de Thébaïde comme Gascou le supposait).

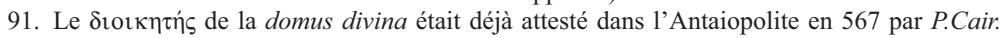
Masp. I 67002 iii, 1 (cette fois comme subordonné du duc), et, dans le présent dossier, par les lettres

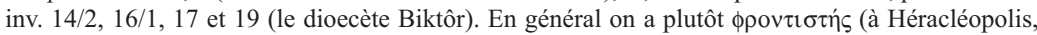
d'après $C P R$ V 18 et $S P P$ III 295) ou $\phi \rho o v \tau i \zeta \omega v$ (ainsi à Oxyrhynchus ou à Mylasa; voir D. Feissel, «L'ordonnance du préfet Dionysios inscrite a Mylasa en Carie (ler août 480) », Travaux et Mémoires 12,1994 , p. 285 , n. 88 ). 


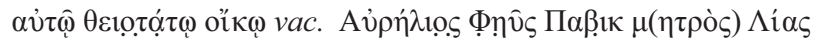

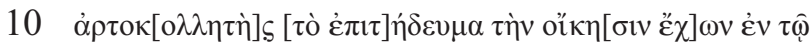

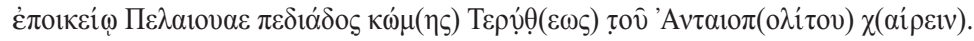

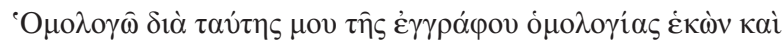

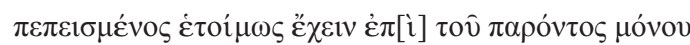

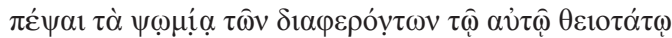

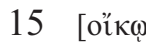
$\pm 14$

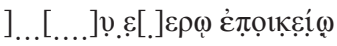

$$
\begin{aligned}
& \text { ] } \kappa \omega \mu(\text { ) } \operatorname{Te\rho v\theta (~)~}
\end{aligned}
$$

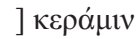

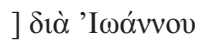

$$
\begin{aligned}
& \pi \alpha \rho] \dot{\varepsilon} \xi \omega \chi \rho v[\sigma] \circ \hat{v}
\end{aligned}
$$

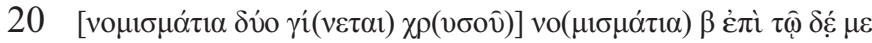

$$
\text { ] } \dot{\alpha} \mu 1 \sigma \theta \varepsilon \dot{c}
$$

(m. 2) A ن̉

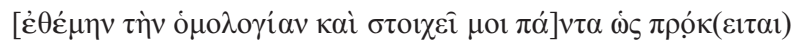

$$
\text { ] } \varepsilon \varphi \varsigma \varsigma \alpha \dot{\alpha} \xi 1 \omega \theta \varepsilon i \varsigma
$$

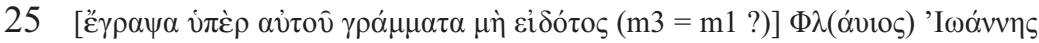

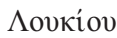

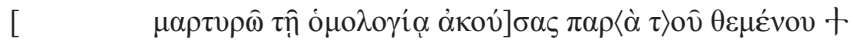

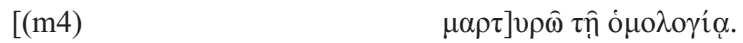

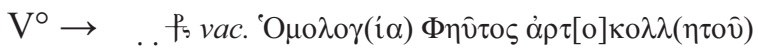

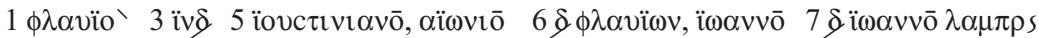

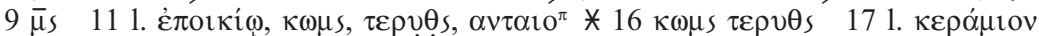

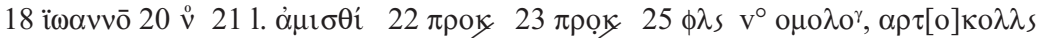

Post-consulat de notre seigneur, Flavius Justinien l'éternel Auguste, empereur, pour la troisième fois, pharmouthi 16, douzième indiction.

À la très divine maison de notre sérénissime seigneur Flavius Justinien l'éternel Auguste, empereur, représentée par les Flavii Horiôn et Iôannès, les très glorieux illustres et arabarques, représentés par toi, Flavius Iôannès, le clarissime dioecète des biens antaiopolites revenant à ladite très divine maison, Aurelius Phèus fils de Pabik, de mère Lia, boulanger de profession, ayant son domicile au hameau de Pelaionae du terroir du village de Terythis de l'Antaiopolite, salut.

Je conviens, par le présent contrat écrit, de bon gré et sincèrement, d'être disposé, pour l'année présente seulement, à boulanger les pains des dépendants de ladite très divine maison [...Je donnerai deux sous d'or ... soit] deux sous d'or, étant entendu que je [...] sans 
recevoir de salaire [... Aurelius] Phèus, le susmentionné, j'ai conclu ce contrat et j'agrée à toutes ses dispositions comme il est stipulé ci-dessus [...Un tel] sur sa demande j'ai écrit pour lui qui ne sait pas les lettres. Flavius Jôannès fils de Loukios [...] je suis témoin du contrat, ayant entendu celui qui l'a conclu [... Un tel ...] je suis témoin du contrat [...].

\section{Au verso : Contrat de Phèus le boulanger.}

9. Le nom $\Phi \eta v ̂ \varsigma$ est bien attesté à l'époque dans l'Antaiopolite et l'Apollonopolite mineur ; le patronyme Pabik est lui aussi connu dans l'Antaiopolite byzantin et arabe. Ce dernier nom pourrait contenir une allusion au faucon ou à la corneille (G. Nachtergael et R. Pintaudi, «Un seul Dieu, celui qui vient en aide », Aegyptus 86,2006, p. 71 , n. text. 3 , à propos d'un nom Abik).

10. La lecture se fonde sur la 1.28 (formule d'endossement $\mathrm{du} \mathrm{v}^{\circ}$ ); la présente désignation du boulanger est, semble-t-il, propre à l'Antaiopolite (voir J.-L. Fournet,

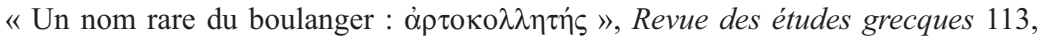
2000, p. 392-412, citant en particulier cette occurrence).

11. Pour la lecture du toponyme Tépv $\theta \imath \varsigma$, voir la 1 . 18. Il était déjà attesté sous

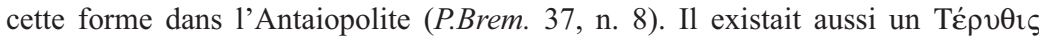
dans l'Apollinopolite Mineur (voir P.Köln III 151, n. 5), ressort limitrophe de l'Antaiopolite. Malgré cette proximité, l'état actuel de la documentation n'autorise pas à les confondre car ce toponyme est attesté dans plusieurs nomes.

14. $\pi \dot{\varepsilon} \psi \alpha \imath$ : le verbe $\pi \dot{\varepsilon} \sigma \sigma \omega / \pi \varepsilon ́ \pi \tau \omega$ (cuire, boulanger) est très peu attesté dans les papyrus (P.Lond. VII 2149, 12), de même que la forme composée $\varepsilon \kappa \pi \varepsilon ́ \sigma \sigma \omega$ de $P$. Alex. 1, 8 et $S B$ XX 15159, 2-3 (fournitures de pain à l'occasion d'une visite de l'empereur Hadrien).

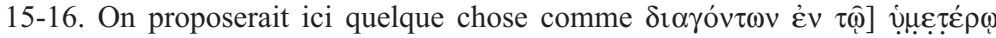

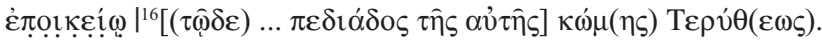

19-21. Ici s'introduisent des clauses pénales au cas où Phèus ne s'acquitterait pas de sa tâche dans les délais requis (versement d'une indemnité de deux sous d'or et engagement à poursuivre le travail sans rémunération, $\alpha \mu \imath \sigma \theta i)$. La graphie ó $\mu \iota \sigma \theta \varepsilon i$ est déjà attestée par $S B$ XX 15027, 5. Les restitutions de la 1.20 , tout en étant nécessaires, sont un peu courtes. Peut-être la somme spécifiée contenait-elle en plus une allusion à l'étalon monétaire local, qui n'aurait pas été reprise dans la totalisation.

26. Les restitutions sont sûres mais trop courtes. Le nom du témoin de la 1.25 devait être suivi, au début de la ligne, d'une qualité professionnelle et/ou d'une origo.

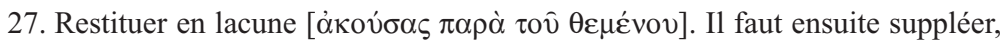
après un vacat, la formule de complétion du tabellion local.

28. Devant le stavrogramme, vestiges de deux signes, le dernier étant suscrit, indice d'une forme abrégée. Dans le vacat qui suit le stavrogramme, on discerne des empreintes d'encre. Pour la qualité professionnelle de l'intéressé, voir n. 10

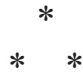

